Sobre a existência ou não de bases normais auto-duais para extensões galoisianas de corpos 
SERVIÇO DE PÓS-GRADUAÇÃO DO ICMC-USP

Data de Depósito:

Assinatura:

\title{
Sobre a existência ou não de bases normais auto-duais para extensões galoisianas de corpos
}

\author{
Sávio da Silva Coutinho
}

Orientadora: Profa. Dra. Ires Dias

Dissertação apresentada ao Instituto de Ciências Matemáticas e de Computação - ICMC-USP, como parte dos requisitos para obtenção do título de Mestre em Matemática

\section{USP - São Carlos}

Janeiro/2009

\footnotetext{
${ }^{1} \mathrm{O}$ autor teve apoio financeiro da CAPES.
} 
Aos meus pais José e Darci, minha irmã, Andréia, e minha noiva, Priscilla. 


\section{Agradecimentos}

Agradeço primeiramente a Deus por ter dado a mim a oportunidade.

Agradeço aos mesu pais, José e Darci, pelo amor incondicional e pelo apoio durante todo esse período e principalmente nos momentos mais difíceis. Agradeço a minha irmã, Andréia, pelo amor, carinho e conselhos nas horas difíceis e por estar sempre à disposição de me ajudar.

Agradeço à minha noiva, Priscilla, pelo amor, carinho, pela compreensão durante todos esses anos e por me animar em momentos difíceis. Agradeço ao Walter pela ajuda dada na digitação.

Agradeço aos professores e colegas do IME e do ICMC e a todos aqueles que foram responsáveis pela minha formação, em especial ao professor Henrique Guzzo Júnior.

Agradeço à minha orientadora, Ires, pela paciência, compreensão e pelos conselhos.

Por fim, agradeço a todos que, de alguma forma, contribuíram para a realização deste trabalho e à CAPES pelo apoio financeiro. 


\section{Resumo}

Neste trabalho, apresentamos um estudo sobre a existência ou não de bases normais auto-duais para extensões galoisianas finitas de corpos, mostrando que toda extensão galoisiana finita de grau ímpar posui uma base normal auto-dual, enquanto que para extensões galoisianas de grau par, apresentamos algumas condições suficientes que garantem a não existência de bases normais auto-duais. 


\section{Abstract}

In this work, we present a study about the existence or not of self-dual normal bases for finite galoisian extensions of fields, showing that all the odd degree finite galoisian extension has a self-dual normal base, whereas for even degree galoisian extensions, we present some sufficient conditions that assure the non-existence of self-dual normal bases. 


\section{Sumário}

1 Preliminares e o teorema da base normal 3

1.1 Extensões galoisianas de corpos . . . . . . . . . . . . . . 3

1.2 Formas bilineares e hermitianas . . . . . . . . . . . . . . 7

1.3 Independência dos caracteres e a forma traço . . . . . . . . . . . . . . . 14

1.4 O teorema da base normal . . . . . . . . . . . . . . . 23

2 Sobre a existência de bases normais auto-duais $\quad 29$

3 Sobre a não existência de bases normais auto-duais 39

$\begin{array}{ll}\text { Referências Bibliográficas } & 55\end{array}$ 



\section{Introdução}

Na teoria de Galois sobre corpos, ou mais precisamente, no estudo de extensões finitas de corpos $L \supseteq K$, é de fundamental importância encontrarmos bases especiais para $L$ sobre $K$. Um tipo de base especial são as chamadas bases normais. Sejam $L \supseteq K$ uma extensão galoisiana de corpos com grau $n$ e grupo de Galois $G=\left\{\sigma_{1}, \sigma_{2}, \ldots, \sigma_{n}\right\}$. Uma base normal de $L$ sobre $K$ é uma base da forma $\mathcal{B}=\left\{\sigma_{1}(a), \sigma_{2}(a), \ldots, \sigma_{n}(a)\right\}$, para algum $a \in L$, chamado o gerador da base normal $\mathcal{B}$. Assim, dizemos que a extensão galoisiana $L \supseteq K$ admite uma base normal se existe um elemento $a \in L$ tal que seus conjugados formam uma base de $L$ sobre $K$.

Também para extensões galoisianas, podemos considerar a forma bilinear traço, induzida pela aplicação traço $\operatorname{Tr}_{L / K}$ de $L$ sobre $K$ e, termos a noção de ortogonalidade, ou seja, uma base $\mathcal{B}^{\prime}=\left\{a_{1}, a_{2}, \ldots, a_{n}\right\}$ de $L$ sobre $K$ é dita ser ortogonal se $T\left(a_{i}, a_{j}\right)=$ 0, para $i \neq j$, onde $T: L \times L \rightarrow K$ é a forma bilinear traço de $L$ sobre $K$.

Neste trabalho, apresentamos um estudo sobre a existência ou não de bases normais auto-duais para extensões galoisianas finitas de corpos, mostrando que toda extensão galoisiana finita de grau ímpar possui uma base normal auto-dual, enquanto que para extensões galoisianas de grau par, apresentamos algumas condições suficientes que garantem a não existência de bases normais auto-duais.

No Capítulo 1, apresentamos conceitos e propriedades básicas necessárias para o desenvolvimento e compreensão dos resultados principais do trabalho, que serão apresentados nos capítulos seguintes, finalizando com a demonstração do Teorema da Base Normal. 
O Capítulo 2, baseado em [2], é dedicado a demonstrar a existência de bases normais auto-duais no caso em que o grau da extensão galoisiana de corpos é ímpar.

Por fim, no Capítulo 3, baseado em [6], mostraremos condições suficientes que garantem a não existência de bases normais auto-duais. 


\section{CAPÍTULO 1}

\section{Preliminares e o teorema da base normal}

Neste primeiro capítulo apresentamos os conceitos e alguns resultados preliminares necessários para o entendimento e desenvolvimento dos dois resultados que serão apresentados nos dois próximos capítulos.

Alguns resultados básicos da teoria de Galois sobre corpos e sobre formas bilineares, serão apresentados sem demonstração que, caso seja do interesse do leitor, podem ser encontradas em [8] e/ou [9].

\subsection{Extensões galoisianas de corpos}

Nesta seção, para facilitar a leitura do restante deste trabalho, apresentamos alguns resultados sobre extensões de corpos e grupos de Galois que fazem parte de qualquer curso básico de teoria de Galois, cujas demonstrações podem ser encontradas, por exemplo em [5] e/ou em [9].

No que segue em todo esse trabalho, denotaremos por $L \supseteq K$ a extensão de corpos $L$ sobre $K$ e, por $[L: K]$ o seu grau. Todas as extensões de corpos consideradas serão assumidas serem finitas, isto é, $[L: K]<\infty$.

O conjunto dos $K$-automorfismos de $L$, isto é, dos automorfismos $\sigma: L \rightarrow L$, tais que $\sigma(x)=x$, para todo $x \in K$, com a operação de composição, forma um grupo, dito ser o grupo de Galois de $L$ sobre $K$ e denotado por $\operatorname{Gal}(L / K)$. 
Se $G$ é um grupo finito, denotamos por $|G|$ o número de elementos de $G$, que é a ordem de $G$. Da mesma forma, se $\sigma \in G$, denotamos por $|[\sigma]|$ a ordem do subgrupo cíclico $[\sigma]$.

Seja $L \supseteq K$ uma extensão de corpos. Um elemento $a \in L$ é dito ser separável sobre $K$ se o seu polinômio minimal sobre $K$ não possuir raízes múltiplas. A extensão $L \supseteq K$ é dita ser uma extensão separável se cada elemento de $L$ for separável sobre $K$.

A extensão $L \supseteq K$ é dita ser uma extensão normal se $L$ for corpo de raízes de algum polinômio $f(X) \in K[X]$, ou seja, $L$ for o menor corpo que contém $K$ e todas as raízes de $f(X)$. O fêcho normal de $L \supseteq K$ é a menor extensão $F$ de $L$ tal que $F \supseteq K$ é normal.

A extensão separável $L \supseteq K$ é dita ser uma extensão de Galois, ou $L$ é dita ser uma extensão galoisiana de $K$, se $[L: K]=|G a l(L / K)|$, ou seja, o grau da extensão $L \supseteq K$ é igual a ordem do grupo de Galois de $L$ sobre $K$.

O próximo resultado apresenta uma caracterização das extensões galoisianas:

Teorema 1.1 Seja $L \supseteq K$ uma extensão de corpos. As seguintes condições são equivalentes:

(a) $L \supseteq K$ é uma extensão galoisiana;

(b) L é corpo de raízes de algum polinômio separável $f(X) \in K[X]$;

(c) L é normal, separável e tem dimensão finita sobre $K$.

Finalizamos esta seção com a apresentação do teorema fundamental da teoria de Galois. Para tanto, considere $L \supseteq K$ uma extensão galoisiana de corpos, com grupo de Galois $G=\operatorname{Gal}(L / K)$.

Sejam $\Delta=\{F$ corpo; $L \supseteq F \supseteq K\}$ o conjunto dos subcorpos intermediários de $L \supseteq K$ e, $\Sigma=\{H \subset G ; H$ é subgrupo de $G\}$, o conjunto dos subgrupos de $G$.

Para cada elemento $H \in \Sigma$, associamos o elemento de $\Delta, L^{H}=\{x \in L ; \sigma(x)=x$, para todo $\sigma \in H$ \}, ou seja, o subcorpo dos elementos de $L$ fixados por $H$. Por outro 
lado, para cada $F \in \Delta$, temos que $L \supseteq F$ é uma extensão galoisiana de corpos e, associamos à $F$ o subgrupo de $G, G a l(L / F)=\{\sigma \in G ; \sigma(x)=x$, para todo $x \in F\}$ o subgrupo dos elementos de $G$ que fixam $F$. Com estas notações, temos:

Teorema 1.2 (Teorema Fundamental de Galois) As aplicações $H \mapsto L^{H}$ e $F \mapsto$ $\operatorname{Gal}(L / F)$, com $H \in \Sigma$ e $F \in \Delta$, são bijeções sendo uma inversa da outra. Além disso, temos que:

(a) Tais aplicações invertem inclusões;

(b) $|H|=\left[L: L^{H}\right] e[G: H]=\left[L^{H}: K\right]$;

(c) H é um subgrupo normal de $G$ se, e somente se $L^{H} \supseteq K$ é uma extensão normal. Neste último caso temos também que $\operatorname{Gal}\left(L^{H} / K\right) \cong G / H$.

Seja $L \supseteq K$ uma extensão de corpos. Dizemos que $L \supseteq K$ é uma extensão simples, ou que $L$ admite um elemento primitivo sobre $K$, se existir $a \in L$ tal que $L=K(a)$, ou seja, $L$ é gerado por $K$ e $a$. Neste caso, se $[L: K]=n$, então $\left\{1, a, a^{2}, \cdots, a^{n-1}\right\}$ é uma base de $L$ sobre $K$, dita ser a base gerada pelo elemento primitivo $a$. Sobre extensões simples temos:

Teorema 1.3 Seja $L \supseteq K$ uma extensão de corpos. Temos que $L \supseteq K$ é uma extensão simples se, e somente se existem um número finito de corpos intermediários entre $L$ e K.

Demonstração: Vamos supor inicialmente que existe $a \in L$ tal que $L=K(a)$. Sejam $F$ um subcorpo de $L$ contendo $K, f(X) \in K[X]$ o polinômio minimal de $a$ sobre $K$ e $g(X) \in F[X]$ o polinômio minimal de $a$ sobre $F$. Então, temos que $g(X) \mid f(X)$ em $F[X]$. Seja $E$ o subcorpo de $L$ gerado por $K$ e pelos coeficientes de $g(X)$. Então, $E \subseteq F$ e claramente o polinômio minimal de a sobre $E$ também é $g(X)$. Como $L=F(a)=E(a)$, temos que $[L: F]=\operatorname{grau}(g(X))=[L: E]$, de onde concluímos 
que $F=E$. Com isso, mostramos que o número de corpos intermediários entre $K$ e $L$ deverá ser finito pois o número de divisores de $g(X)$ é finito.

Reciprocamente, vamos supor agora que $L \supseteq K$ possui um número finito de corpos intermediários. Se $K$ é finito, então $L$ também será finito, já que $|L|=|K|^{[L: K]}$. Pelo fato de $L$ ser um corpo finito, sabemos que o grupo multiplicativo ( $\dot{L}=L-\{0\},$.$) é um$ grupo cíclico e portanto existe $a \in \dot{L}$ tal que $\dot{L}=[a]$, logo $L=K(a)$, ou seja, a extensão $L \supseteq K$ é simples. Podemos então assumir que $K$ é infinito. Como $[L: K]<\infty$, temos que $L=K\left(a_{1}, \cdots, a_{n}\right)$, para alguns $a_{1}, \cdots, a_{n} \in L$, ou seja, $L$ é gerada por $K$ e $\left\{a_{1}, \cdots, a_{n}\right\}$. Agora, usando indução sobre $n$, é suficiente mostrarmos o caso em que $n=2$, ou seja, basta mostrarmos que para dois elementos quaisquer $a, b \in L$ a extensão $K(a, b) \supseteq K$ é simples. Consideremos os subcorpos $K(a+c b) \subseteq K(a, b)$, onde $c \in K$. Por hipótese temos que existe somente um número finito de tais corpos, e, como $K$ é infinito existem $c \neq d$ em $K$ tais que $K(a+c b)=K(a+d b)$. Logo, $b=(c-d)^{-1}(a+c d-(a-d b)) \in K(a+c b)$, o que mostra que $K(a, b)=K(a+c b)$, ou seja, $a+c b$ é um elemento primitivo de $K(a, b)$, como queríamos demonstrar.

Como conseqüência deste teorema, temos o teorema do elemento primitivo.

Corolário 1.4 Toda extensão finita e separável de corpos admite um elemento primitivo.

Demonstração: Sejam $L \supseteq K$ uma extensão finita e separável e $F \supseteq K$ seu fêcho normal. Desde que $F \supseteq K$ é finita, se mostrarmos que $F \supseteq K$ é uma extensão separável, então teremos que $F$ é uma extensão galoisiana de $K$. Para isso, observemos que todo elemento de $F$ é raiz do polinômio minimal de algum elemento de $L$. Como $L \supseteq K$ é separável, temos que o polinômio minimal de todo elemento de $L$ é separável sobre $K$. Assim, todo elemento de $F$ é separável sobre $K$. Logo, $F \supseteq K$ é galoisiana.

Notemos que corpos intermediários de $L \supseteq K$ são também corpos intermediários de $F \supseteq K$. Como existe apenas uma quantidade finita de subgrupos do grupo $\operatorname{Gal}(F / K)$, 
o teorema 1.2 (teorema fundamental da teoria de Galois), nos garante que existe apenas uma quantidade finita de corpos intermediários de $F \supseteq K$. Conseqüentemente, $L \supseteq K$ tem somente uma quantidade finita de corpos intermediários, o que implica, do teorema anterior, que $L \supseteq K$ admite um elemento primitivo.

\subsection{Formas bilineares e hermitianas}

Nesta seção, começaremos apresentando, a noção de forma e/ou módulo hermitiano sobre um anel com elemento identidade. Depois, apresentaremos as noções básicas sobre formas bilineares e alguns resultados sobre anéis de Witt dos espaços bilineares sobre corpos, que serão importantes para demonstrarmos a existência de bases normais auto-duais para extensões galoisianas de grau ímpar de corpos.

No que segue, $A$ denotará um anel com elemento unidade 1 , todos os $A$-módulos considerados, a menos de menção contrária, serão $A$-módulos à esquerda unitários.

Uma involução sobre o anel $A$ é uma aplicação - : $A \rightarrow A$, tal que para todo $a, b \in A$, temos:

(a) $\overline{a+b}=\bar{a}+\bar{b}$;

(b) $\overline{a b}=\bar{b} \cdot \bar{a}$;

(c) $\overline{\bar{a}}=a$,

ou seja, uma involução sobre $A$ é um anti-automorfismo de $A$ de ordem 2 .

Observe que uma involução - $: A \rightarrow A$ é sempre bijetora, pois de (c) temos a sobrejeção e se $\bar{a}=\bar{b}$, então $a=\overline{\bar{a}}=\overline{\bar{b}}=b$.

Sejam - $: A \rightarrow A$ uma involução e $M$ um $A$-módulo. Uma forma sesquilinear sobre $M$ é uma aplicação $s: M \times M \rightarrow A$ tal que

(a) $s(x+y, z)=s(x, z)+s(y, z)$;

(b) $s(x, y+z)=s(x, y)+s(x, z)$;

(c) $s(a x, b y)=a s(x, y) \bar{b}$

para quaisquer que sejam $a, b \in A$ e $x, y \in M$. 
Uma forma hermitiana sobre $M$ é uma forma sesquilinear $h: M \times M \rightarrow A$ que satisfaz $h(x, y)=\overline{h(y, x)}$, para todo $x, y \in M$. O par $(M, h)$ é dito ser um módulo hermitiano sobre $A$. Um módulo hermitiano $(M, h)$ é dito ser hermitiano par se existir uma forma sesquilinear $s: M \times M \rightarrow A \operatorname{com} h(x, y)=s(x, y)+\overline{s(y, x)}$, para todo $x, y \in M$.

O próximo resultado mostra que sobre certas condições, todo módulo hermitiano sobre o anel $A$ é par.

Lema 1.5 Seja $Z(A)$ o centro do anel $A$. Se existe $a \in Z(A)$, tal que $a+\bar{a}=1$, então todo módulo hermitiano sobre A é par.

Demonstração: Sejam $(M, h)$ um módulo hermitiano sobre $A$ e $a \in Z(A)$, com $a+\bar{a}=$ 1. Consideremos $s: M \times M \rightarrow A$, definida por $s(x, y)=a h(x, y)$, para todo $x, y \in M$. Temos que $s$ é uma forma sesquilinear sobre $M$. Agora, para todo $x, y \in M$, temos $h(x, y)=1 h(x, y)=(a+\bar{a}) h(x, y)=a h(x, y)+\bar{a} h(x, y)=a h(x, y)+\bar{a} \overline{h(y, x)}$.

Note que $\overline{s(y, x)}=\overline{a h(y, x)}=\bar{a} \overline{h(y, x)}$, pois $a \in Z(A)$. Conseqüentemente,

$$
h(x, y)=a h(x, y)+\bar{a} \overline{h(y, x)}=s(x, y)+\overline{s(y, x)},
$$

o que mostra que $(M, h)$ é par.

Observação 1.6 Se $K$ é um corpo de característica distinta de $2, \operatorname{Car}(K) \neq 2$, então todo módulo hermitiano sobre $K$ é par. De fato, considerando sobre $K$ a involução trivial, temos que $a=\frac{1}{2} \in Z(K)$ satisfaz o lema 1.5 .

Dado $a \in A, \operatorname{com} \bar{a}=a$, denotaremos por $\langle a\rangle: A \times A \rightarrow A$ a forma hermitiana de posto 1 , definida por $(r, s) \mapsto r a \bar{s}$, para todo $r, s \in A$.

Sejam $M$ um $A$-módulo e $M^{*}=\operatorname{Hom}_{R}(M, A)$ o seu dual, que tem uma estrutura de $A$-módulo à esquerda dada por $(a f)(x)=f(x) \bar{a}$, para todo $a \in A, f \in M^{*}$ e $x \in M$.

Se $(M, h)$ é um módulo hermitiano sobre $A$, então a aplicação $A$-linear $H: M \rightarrow M^{*}$, dada por $H(x)(y)=h(y, x)$, para todo $x, y \in M$, é chamada a aplicação adjunta de $h$. 
Dizemos que a forma hermitiana $h$ é não-singular, ou que o módulo hermitiano $(M, h)$ é não-singular, se a aplicação adjunta é um isomorfismo de $A$-módulos à esquerda. Note que a forma hermitiana $\langle a\rangle$ é não-singular se, e somente se $a \in \dot{A}$, onde $\dot{A}$ denota o grupo dos elementos inversíveis do anel $A$.

Vejamos agora algumas noções básicas da teoria algébrica das formas bilineares sobre corpos. A definição de formas e/ou espaços bilineares e a construção do anel de Witt dos espaços bilineares, sobre corpos, serão omitidos aqui e, podem ser encontrados em [11].

Sejam $L \supseteq K$ uma extensão de corpos, $V$ um espaço vetorial sobre $L, s: L \rightarrow K$ uma função $K$-linear não nula e $b: V \times V \rightarrow L$ uma forma bilinear simétrica sobre $L$. Notemos que $V$ é também um $K$-espaço vetorial e a composta $s b: V \times V \rightarrow K$, é uma forma bilinear simétrica sobre $K$. Mais ainda, se $x, y \in V$ são ortogonais no $L$-espaço bilinear $(V, b)$, ou seja, $b(x, y)=0$, então $x$ e $y$ também serão ortogonais no $K$-espaço bilinear $(V, s b)$.

O $K$-espaço bilinear $(V, s b)$ é chamado o transfer de $(V, b)$ e denotado por $s^{*}(V, b)$. Pode-se mostrar que $s^{*}$ leva $L$-espaços hiperbólicos em $K$-espaços hiperbólicos e que $s^{*}$ induz um homomorfismo de grupos $s^{*}: W(L) \rightarrow W(K)$, com $(V, b) \mapsto(V, s b)$, onde $W(L)$ e $W(K)$ são os anéis de Witt dos espaços bilineares sobre $L$ e $K$ respectivamente e, denotamos também por $(V, b)$ a classe do espaço bilinear $(V, b)$ no anel de Witt.

Sejam $x \in L$ e $G=\operatorname{Gal}(L / K)=\left\{\sigma_{1}=i d, \sigma_{2}, \ldots, \sigma_{n}\right\}$. A norma de $L$ sobre $K$ de $x$ é definida por $N_{L / K}(x)=\prod_{i=1}^{n} \sigma_{i}(x)$. Se $m(X)=X^{r}+a_{r-1} X^{r-1}+\cdots+a_{0}$ é o polinômio minimal de $x$ sobre $K$, então $N_{L / K}(x)=(-1)^{r} a_{0}$. De fato, se $u_{1}, \cdots, u_{r}$ são as raízes do polinômio minimal, então $m(X)=\left(X-u_{1}\right) \cdots\left(X-u_{r}\right)=X^{r}+a_{r-1} X^{r-1}+$ $\cdots+a_{0}$, conseqüentemente $a_{0}=(-1)^{r} \prod_{i=1}^{r} u_{i}$. Observe que para $\sigma \in \operatorname{Gal}(L / K)$ temos que $\sigma(m(X))=m(X)$. Portanto $a_{0}=\sigma\left(a_{0}\right)=\sigma\left((-1)^{r} \prod_{i=1}^{r} u_{i}\right)=(-1)^{r} \prod_{i=1}^{r} \sigma\left(u_{i}\right)=$ $(-1)^{r} \prod_{i=1}^{r} \sigma_{i}(x)=(-1)^{r} N_{L / K}(x)$, ou seja, $N_{L / K}(x)=(-1)^{r} a_{0}$. 
Para $a \in L$, denotamos por $\langle a\rangle$ o espaço bilinear $\langle a\rangle: L \times L \rightarrow L$, definido por $\langle a\rangle(x, y)=x a y$, para todo $x, y \in L$. Nos próximos resultados, utilizaremos o símbolo $\simeq$ para denotar que duas formas bilineares são isométricas.

O próximo resultado, devido à Scharlau, mostra como é a imagem do espaço $\langle 1\rangle$ em $W(L)$ para uma extensão simples $L \supseteq K$ e um particular transfer $s^{*}$.

Teorema 1.7 Seja $L=K(a) \supseteq K$ uma extensão simples com $[L: K]=n$ e $s: L \rightarrow K$ uma aplicação K-linear, definida por $s(1)=1, s(a)=\cdots=s\left(a^{n-1}\right)=0$. Então,

(a) Se $n=2 m$, temos $s^{*}\left(\langle 1\rangle_{L}\right) \simeq(m-1) \mathbb{H}_{K} \perp\left\langle 1,-N_{L / K}(a)\right\rangle$, onde $\mathbb{H}_{K}$ denota o plano hiperbólico $\langle 1,-1\rangle$ sobre $K$ e $\langle 1\rangle_{L}$ é o espaço bilinear $\langle 1\rangle$ sobre $L$;

(b) Se $n=2 m+1$, temos $s^{*}\left(\langle 1\rangle_{L}\right) \simeq m \mathbb{H}_{K} \perp\langle 1\rangle_{K}$, onde $\langle 1\rangle_{K}$ é o espaço bilinear 〈1〉 sobre $K$.

Demonstração: Note que $\left\{1, a, a^{2}, \ldots, a^{n-1}\right\}$ é uma base de $L$ sobre $K$ e $s^{*}\left(\langle 1\rangle_{L}\right)(x, y)$ $=s(x y)$, para todo $x, y \in L$.

Seja $L_{0}=K a+K a^{2}+\ldots+K a^{n-1} \subseteq L$. Com relação a forma bilinear $s^{*}\left(\langle 1\rangle_{L}\right)$, temos que os subespaços $K$ e $L_{0}$ são ortogonais. De fato, para todo $x \in K$ e $y=$ $y_{1} a+\ldots+y_{n-1} a^{n-1} \in L_{0}$, temos

$$
s^{*}\left(\langle 1\rangle_{L}\right)(x, y)=s(x y)=y_{1} x s(a)+\ldots+y_{n} x s\left(a^{n-1}\right)=0 .
$$

Assim, temos que $s^{*}\left(\langle 1\rangle_{L}\right)=\langle 1\rangle_{K} \perp L_{0}$.

Agora, analisemos separadamente os casos em que $n=[L: K]$ é par e ímpar.

Se $n=2 m$, então $\operatorname{dim}_{K} L_{0}=2 m-1$ e $\left\{a, a^{2}, \ldots, a^{m-1}\right\}$ gera um subespaço totalmente isotrópico de $L_{0}$, pois $s^{*}\left(\langle 1\rangle_{L}\right)\left(a^{i}, a^{j}\right)=s\left(a^{i+j}\right)=0$, para todo $i, j=1, \ldots, m-1$. Por um resultado clássico sobre formas bilineares (ver teorema 4.5 em [11]), temos que $L_{0}$ contém um subespaço hiperbólico de dimensão $2(m-1)$, que é isométrico a uma soma de $(m-1)$ planos hiperbólicos $\mathbb{H}_{K}$, de onde obtemos que $L_{0} \simeq(m-1) \mathbb{H}_{K} \perp L_{1}$, com $\operatorname{dim}_{K} L_{1}=1$. Resta determinarmos a forma bilinear restrita ao subespaço $L_{1}$. 
Para tanto, consideremos a matriz da forma bilinear $s_{*}\left(\langle 1\rangle_{L}\right)$ restrita a $L_{0}$, com relação a base $\left\{a, a^{2}, \ldots, a^{n-1}\right\}$.

$$
M=\left[\begin{array}{ccccc}
s\left(a^{2}\right) & s\left(a^{3}\right) & \cdots & s\left(a^{n-1}\right) & s\left(a^{n}\right) \\
s\left(a^{3}\right) & s\left(a^{4}\right) & \cdots & s\left(a^{n}\right) & s(a) \\
\vdots & \vdots & \ddots & \vdots & \vdots \\
s\left(a^{n}\right) & s\left(a^{n-1}\right) & \cdots & s\left(a^{n-3}\right) & s\left(a^{n-2}\right)
\end{array}\right]
$$

Como $s\left(a^{i}\right)=0$, para $i=1, \ldots, n-1$, resta calcularmos $s\left(a^{n}\right)$. Como $L=K(a)$ e $[L: K]=n$, temos que o polinômio minimal de $a$ sobre $K$ é da forma $X^{n}+c_{n-1} X^{n-1}+$ $\cdots+c_{1} X+c_{0}$. De onde segue que $a^{n}=-c_{n-1} a^{n+1}-\cdots-c_{1} a-c_{0}$ e, como $s$ é $K$-linear, obtemos $s\left(a^{n}\right)=-c_{0}$.

Calculando os determinantes, temos $\operatorname{det}\left(L_{0}\right)=\operatorname{det}(M)=(-1)^{m} c_{0}^{n-1}=(-1)^{m} c_{0}^{n-1}$. Por outro lado, $L_{0} \simeq(m-1) \mathbb{H}_{K} \perp L_{1}$, então

$$
(-1)^{m} c_{0}^{n-1}=\operatorname{det}\left(L_{0}\right)=\operatorname{det}\left((m-1) \mathbb{H}_{K} \perp L_{1}\right)=(-1)^{m-1} \operatorname{det}\left(L_{1}\right),
$$

o que implica que $\operatorname{det}\left(L_{1}\right)=-c_{0}^{2 m-1}=-c_{0} \dot{K}^{2}$.

Mais ainda, como o polinômio minimal de $a$ é $X^{n}+c_{n-1} X^{n-1}+\cdots+c_{0}$, temos que $N_{L / K}(a)=(-1)^{n} c_{0}=c_{0}$. Assim, quando $n=2 m$, temos

$$
s^{*}\left(\langle 1\rangle_{L}\right) \simeq\langle 1\rangle_{K} \perp L_{0} \simeq(m-1) \mathbb{H}_{K} \perp\left\langle 1,-N_{L / K}(a)\right\rangle,
$$

como queríamos.

Se $n$ é ímpar, então $n=2 m+1$, então $\operatorname{dim} L_{0}=n-1=2 m$ e, como visto acima obtemos que $\left\{a, a^{2}, \ldots, a^{m}\right\}$ gera um subespaço totalmente isotrópico de $L_{0}$ com dimensão $m$, o que mostra que $L_{0} \cong m \mathbb{H}_{K}$ e, neste caso, $s^{*}\left(\langle 1\rangle_{L}\right) \simeq\langle 1\rangle_{K} \perp L_{0} \simeq$ $\langle 1\rangle_{K} \perp m \mathbb{H}_{K}$, o que mostra o teorema.

No que segue, $K$ denotará um corpo cuja característica é diferente de $2, A$ uma $K$-álgebra de dimensão finita e - uma involução $K$-linear sobre $A$.

Se $(M, h)$ é um módulo hermitiano não-singular sobre $A$ e se $(V, b)$ é um espaço bilinear não-singular sobre $K$, então o produto $b \otimes h$ é uma forma hermitiana sobre 
$A$, definida no $A$-módulo $M \otimes_{K} V$. De fato, como $\operatorname{Car}(K) \neq 2$, temos que toda forma bilinear simétrica sobre $K$ é da forma $\left\langle a_{1}, \ldots, a_{n}\right\rangle$, ou seja, $V$ tem uma base $\left\{v_{1}, \ldots, v_{n}\right\}$, ortogonal com respeito a forma $b$, isto é, com $b\left(v_{i}, v_{j}\right)=0$ se $i \neq j$ e $b\left(v_{i}, v_{i}\right)=a_{i}$.

Assim, $b=\left\langle a_{1}, \ldots, a_{n}\right\rangle$ e,

$$
\begin{aligned}
& \left\langle a_{1}, \ldots, a_{n}\right\rangle \otimes_{K} h=\left(\left\langle a_{1}\right\rangle \perp \cdots \perp\left\langle a_{n}\right\rangle\right) \otimes_{K} h= \\
& \left\langle a_{1}\right\rangle \otimes_{K} h \perp\left\langle a_{2}\right\rangle \otimes_{K} h \perp \cdots \perp\left\langle a_{n}\right\rangle \otimes_{K} h \simeq a_{1} h \perp \cdots \perp a_{n} h .
\end{aligned}
$$

Esta multiplicação, induz uma estrutura de $W(K)$-módulo no anel de Witt dos espaços hermitianos sobre $A, W(A)$.

Se $L \supseteq K$ é uma extensão de corpos, então podemos estender a involução - de $A$ para uma involução $\bar{L}$ de $A_{L}=A \otimes_{K} L$, com $\overline{a \otimes x}^{L}=\bar{a} \otimes x$, para todo $a \in A$ e $x \in L$.

Para cada módulo hermitiano $(M, h)$ sobre $A$ a extensão de $h$ à $M_{L}=M \otimes_{K} L$ é o $\operatorname{par}\left(M_{L}, h_{L}\right)$, onde

$$
\begin{aligned}
h_{L}: M_{L} \times M_{L} & \rightarrow A_{L} \\
\left(x \otimes c, y \otimes c^{\prime}\right) & \mapsto h(x, y) \otimes c c^{\prime} .
\end{aligned}
$$

Esta extensão induz um homomorfismo de anéis

$$
\begin{aligned}
r^{*}: W(A) & \rightarrow W\left(A_{L}\right) \\
(M, h) & \mapsto\left(M_{L}, h_{L}\right)=(M, h) \otimes_{K} L
\end{aligned}
$$

Seja $s: L \rightarrow K$ uma transformação $K$-linear. Estendemos $s$ a um homomorfismo $A$-linear $s_{A}: A_{L} \rightarrow A$, onde $s_{A}(a \otimes x)=a s(x)$, para todo $a \in A$ e $x \in L$ e obtemos um homomorfismo de grupos

$$
\begin{aligned}
s_{*}: W\left(A_{L}\right) & \rightarrow W(A) \\
(M, h) & \mapsto\left(M, s_{A} h\right) .
\end{aligned}
$$

Considerando o homomorfismo análogo $s_{*}: W(L) \rightarrow W(K)$, temos

$$
s_{*}\left(b \otimes r^{*}(h)\right)=s_{*}(b) \otimes h \quad \text { em } W(A),
$$

para todo $b \in W(L)$ e $h \in W(A)$. 
Para mostrarmos esta última igualdade, devemos mostrar que a aplicação $K$-linear

$$
\begin{aligned}
\Phi: V \otimes_{L}\left(M \otimes_{K} L\right) & \rightarrow V \otimes_{K} M \\
x \otimes_{L}(y \otimes c) & \mapsto c x \otimes y
\end{aligned}
$$

é uma isometria. De fato, para todo $x, x^{\prime} \in V, y, y^{\prime} \in M$ e $c, c^{\prime} \in L$, temos

$$
\begin{aligned}
& s_{*}\left(b \otimes_{L} r^{*}(h)\right)\left(x \otimes_{L}(y \otimes c), x^{\prime} \otimes_{L}\left(y^{\prime} \otimes c^{\prime}\right)\right)= \\
& \left(s_{A}\left(b \otimes_{L} h_{L}\right)\right)\left(x \otimes_{L}(y \otimes c), x^{\prime} \otimes_{L}\left(y^{\prime} \otimes c^{\prime}\right)\right)= \\
& s_{A}\left(\left(b\left(x, x^{\prime}\right) \otimes_{L} h_{L}\left(y \otimes c, y^{\prime} \otimes c^{\prime}\right)\right)=\right. \\
& \left.s_{A}\left(b\left(x, x^{\prime}\right) c c^{\prime} h\left(y, y^{\prime}\right)\right)=s_{A}\left(b\left(c x, c^{\prime} x\right)\right) h\left(y, y^{\prime}\right)\right)= \\
& \left(s_{A} b \otimes h\right)\left(c x \otimes y, c^{\prime} x^{\prime} \otimes y^{\prime}\right)= \\
& s_{*}(b) \otimes h\left(\Phi\left(x \otimes(y \otimes c), x^{\prime} \otimes\left(y^{\prime} \otimes c^{\prime}\right)\right) .\right.
\end{aligned}
$$

Com estas notações temos:

Teorema 1.8 Sejam $L \supseteq K$ uma extensão de corpos de grau ímpar e $A$ uma $K$-álgebra de dimensão finita. Então o homomorfismo $r^{*}: W(A) \rightarrow W\left(A_{L}\right)$, definido acima, é injetor.

Demonstração: Basta mostrarmos o resultado no caso em que $L$ é uma extensão simples de $K$, pois como $[L: K]$ é ímpar, temos que o grau das extensões intermediárias também são ímpares, e, o caso geral seguirá de maneira recursiva.

Desta forma, sejam $L=K(a)$ e $s: L \rightarrow K$ a aplicação $K$-linear, definida por $s(1)=1, s(a)=s\left(a^{2}\right)=\cdots=s\left(a^{n-1}\right)=0$, onde $n=[L: K]$. Tomando $b=\langle 1\rangle_{L}$ em $(\star)$, temos $s_{*}\left(\langle 1\rangle_{L} \otimes r^{*}(h)\right)=s_{*}\left(\langle 1\rangle_{L}\right) \otimes h$ em $W(A)$. Como $\langle 1\rangle_{L} \otimes r^{*}(h)=r^{*}(h) \mathrm{e}$ $[L: K]$ é ímpar, temos do teorema 1.7 que $s_{*}\left(\langle 1\rangle_{L}\right) \otimes h=\langle 1\rangle_{L} \otimes h=h$ em $W(A)$, o que mostra que $s_{*}\left(r^{*}(h)\right)=h$, para todo $h \in W(A)$ e, portanto, $r^{*}$ é injetora.

O próximo resultado o teorema do cancelamento de Witt para formas hermitianas pares, é muito conhecido na teoria algébrica das formas hermitianas. Sua demonstração envolve conceitos que estão além dos nossos objetivos, e por essa razão deixaremos apenas sua referência. 
Teorema 1.9 Seja A uma K-álgebra de dimensão finita. Sejam $(M, h),\left(M^{\prime}, h^{\prime}\right)$ e $(N, g)$ módulos hermitianos pares, não-singulares, sobre A. Se

$$
(M, h) \perp(N, g) \simeq\left(M^{\prime}, h^{\prime}\right) \perp(N, g),
$$

então $(M, h) \simeq\left(M^{\prime}, h^{\prime}\right)$.

Demonstração: Ver teorema 9.1 do Capítulo 7 da referência [11].

Usando o teorema do cancelamento de Witt para módulos hermitianos pares, obtemos um refinamento do teorema 1.9.

Teorema 1.10 Sejam A uma $K$-álgebra de dimensão finita, e $L \supseteq K$ uma extensão de corpos de grau ímpar e $(M, h),\left(M^{\prime}, h^{\prime}\right)$ A-módulos hermitianos pares não-singulares. Se $\left(M_{L}, h_{L}\right) \simeq\left(M_{L}^{\prime}, h_{L}^{\prime}\right)$, então $(M, h) \simeq\left(M^{\prime}, h^{\prime}\right)$.

Demonstração: Como $\left(M_{L}, h_{L}\right) \simeq\left(M_{L}^{\prime}, h_{L}^{\prime}\right)$, temos que eles representam o mesmo elemento em $W\left(A_{L}\right)$. Como $r^{*}$ é injetora, temos que $(M, h)$ e $\left(M^{\prime}, h^{\prime}\right)$ são iguais em $W(A)$. Logo, existem $A$-módulos hermitianos hiperbólicos $(N, g)$ e $\left(N^{\prime}, g^{\prime}\right)$ tais que

$$
(M, h) \perp(N, g) \simeq\left(M^{\prime}, h^{\prime}\right) \perp\left(N^{\prime}, g^{\prime}\right)
$$

Assim, $M \oplus N \cong M^{\prime} \oplus N^{\prime}$ como $A$-módulos e, mais ainda, $M \cong M^{\prime} \operatorname{como} A$ módulos, pois $M_{L} \cong M_{L}^{\prime}$ como $A_{L}$-módulos. Pelo teorema de Krull-Schmidt $(6.4 \mathrm{em}$ [10]) obtemos que $N \cong N^{\prime}$, como $A$-módulos.

Logo, $(N, g) \cong\left(N^{\prime}, g^{\prime}\right)$ como $A$-módulos hermitianos, pois módulos hermitianos hiperbólicos sobre módulos isomorfos são isomorfos (ver 4.10.1 em [1]). Agora, usando o teorema do cancelamento de Witt para módulos hermitianos pares, obtemos que $(M, h) \cong\left(M^{\prime}, h^{\prime}\right)$, como queríamos.

\subsection{Independência dos caracteres e a forma traço}

Nesta seção apresentamos o teorema de independência dos caracteres de Dedeking, um resultado sobre extensões galoisianas, a noção de forma traço de uma extensão 
galoisiana de corpos e alguns resultados básicos sobre o comportamento de tais formas traços, que serão úteis no desenvolvimento dos outros capítulos.

Um caracter de um monóide $H$ em um corpo $F$ é um morfismo de $H$ no grupo multiplicativo $\dot{F}$.

Teorema 1.11 (Teorema de independência de Dedeking) Sejam F um corpo, $H$ um monóide e $\sigma_{1}, \ldots, \sigma_{n}$ caracteres distintos de $H$ em $F$. Então os únicos elementos $a_{1}, \ldots, a_{n}$ em $F$ tais que

$$
a_{1} \sigma_{1}(\phi)+a_{2} \sigma_{2}(\phi)+\cdots+a_{n} \sigma_{n}(\phi)=0
$$

para todo $\phi \in H$, são $a_{1}=a_{2}=\cdots=a_{n}=0$, ou seja, os caracteres $\sigma_{1}, \ldots \sigma_{n}$ são linearmente independentes sobre F.

Demonstração: Mostraremos este resultado por indução sobre $n$. Se $n=1$, então $a_{1} \sigma_{1}(\phi)=0$, para todo $\phi \in H$. Se $a_{1} \neq 0$ teremos que $\sigma_{1}(\phi)=0$, para todo $\phi \in H$, o que nos dá um absurdo, pois $\sigma$ leva elemento neutro em elemento neutro, ou seja, $\sigma\left(1_{A}\right)=1_{F} \neq 0$. Portanto, $a_{1}=0$.

Suponhamos que $n>1$ e que o teorema é verdadeiro para $n-1$ caracteres. Se

$$
a_{1} \sigma_{1}(\phi)+a_{2} \sigma_{2}(\phi)+\cdots+a_{n} \sigma_{n}(\phi)=0
$$

para todo $\phi \in H$, como o teorema é válido para $n-1$ caracteres, podemos supor que $a_{1}, \ldots, a_{n}$ são todos não nulos. Como $\sigma_{1} \neq \sigma_{2}$, existe $\phi_{0} \in H$ tal que $\sigma_{1}\left(\phi_{0}\right) \neq \sigma_{2}\left(\phi_{0}\right)$. Substituindo $\phi$ por $\phi_{0} \phi$ em $(\star)$, obtemos

$$
a_{1} \sigma_{1}\left(\phi_{0}\right) \sigma_{1}(\phi)+a_{2} \sigma_{2}\left(\phi_{0}\right) \sigma_{2}(\phi)+\cdots+a_{n} \sigma_{n}\left(\phi_{0}\right) \sigma_{n}(\phi)=0
$$

Por outro lado, multiplicando os dois lados da igualdade $(\star)$ por $\sigma_{1}\left(\phi_{0}\right)$ obtemos

$$
a_{1} \sigma_{1}\left(\phi_{0}\right) \sigma_{1}(\phi)+a_{2} \sigma_{1}\left(\phi_{0}\right) \sigma_{2}(\phi)+\ldots+a_{n} \sigma_{1}\left(\phi_{0}\right) \sigma_{n}(\phi)=0
$$

Subtraindo estas duas últimas igualdades temos que

$$
b_{2} \sigma_{2}(\phi)+\cdots+b_{n} \sigma_{n}(\phi)=0
$$


onde $b_{i}=a_{i}\left(\sigma_{i}\left(\phi_{0}\right)-\sigma_{1}\left(\phi_{0}\right)\right)$, com $2 \leq i \leq n$.

Por hipótese de indução temos que $b_{i}=0$, para todo $i=2, \cdots, n$, o que contradiz o fato de que $b_{2}=a_{2}\left(\sigma_{1}\left(\phi_{0}\right)-\sigma_{2}\left(\phi_{0}\right)\right) \neq 0$. Portanto, o resultado segue.

Corolário 1.12 Sejam $L$ e $F$ corpos e $\sigma_{1}, \sigma_{2}, \ldots, \sigma_{n}$ imersões distintas de $L$ em $F$. Então $\sigma_{1}, \sigma_{2}, \ldots, \sigma_{n}$ são linearmente independentes sobre $F$.

Demonstração: Claramente as restrições de $\sigma_{i}$ para $\dot{L}$ são caracteres do grupo multiplicativo $H=\dot{L}$ em $F$, e o resultado segue do teorema anterior.

Nosso próximo objetivo é apresentarmos um resultado sobre extensão de corpos e extensões de escalares que usaremos nos próximos capítulos. Para tanto, consideremos $L \supseteq K$ uma extensão de corpos e $G=\operatorname{Gal}(L / K)$ o seu grupo de Galois. Seja $L^{G}$ o subcorpo de $L$ fixado por $G$, isto é, $L^{G}=\{a \in L ; \sigma(a)=a$, para todo $\sigma \in G\}$.

Seja $V$ um $L$-espaço vetorial de dimensão $|G|$ com base $\left\{u_{\sigma} ; \sigma \in G\right\}$. Em $V$ definimos o seguinte produto

$$
\left(\sum_{\sigma \in G} a_{\sigma} u_{\sigma}\right)\left(\sum_{\tau \in G} b_{\tau} u_{\tau}\right)=\sum_{\sigma, \tau \in G} a_{\sigma} \sigma\left(b_{\tau}\right) u_{\sigma \tau}
$$

para todo $a_{\sigma}, b_{\tau} \in L$ e $\sigma, \tau \in G$.

Para todo $a \in L^{G}$ e $x, y \in V$, temos que $(a x) y=x(a y)=a(x y)$. Com este produto e as operações de espaço vetorial de $V$, temos que $V$ é uma $L^{G}$-álgebra. Como $K \subseteq L^{G}$, temos também que $V$ é uma $K$-álgebra. Considere $\operatorname{End}_{K}(L)=\{\phi: L \rightarrow L$; $\phi$ é $K$-linear\}. A aplicação $\Psi: V \rightarrow \operatorname{End}_{K}(L)$, definida por

$$
\Psi\left(\sum_{\sigma \in G} a_{\sigma} u_{\sigma}\right)=\sum_{\sigma \in G} a_{\sigma} \sigma
$$

é um homorfismo de $K$-álgebras que é $L$-linear. Com tais nomenclaturas temos:

Teorema 1.13 Sejam $L \supseteq K$ uma extensão galoisiana de corpos e $G=\operatorname{Gal}(L / K)$. Então a aplicação $\varphi: L \otimes_{K} L \rightarrow L^{|G|}$, dada por $\varphi(x \otimes y)=(x \sigma(y))_{\sigma \in G}$, é um isomorfismo de L-álgebras. 
Demonstração: Como $L \supseteq K$ é galoisiana, temos que $[L: K]=|G|$. Utilizando o teorema 1.11 (independência de Dedeking) é fácil ver que $\Psi$, como definida acima, é uma aplicação injetora. Observe também que $\operatorname{dim}_{K} V=\operatorname{dim}_{L} V \cdot \operatorname{dim}_{K} L=|G| \cdot \operatorname{dim}_{K} L=$ $\left(\operatorname{dim}_{K} L\right)^{2}$. Sabemos também que $\operatorname{dim}_{K}\left(\operatorname{End}_{K}(L)\right)=\left(\operatorname{dim}_{K} L\right)^{2}$, o que mostra que, $\Psi$ é um isomorfismo de $K$-álgebras.

Como $V$ é uma $K$-álgebra, podemos considerar $V$-módulos. Mostraremos agora que para todo $V$-módulo à esquerda $M$ a aplicação $\mu: L \otimes_{K} M^{G} \rightarrow M$, dada por $\mu(x \otimes m)=x m$, é um isomorfismo de $L$-espaços vetoriais, onde $M^{G}=\{y \in M ; \quad \sigma . y=y$ para todo $\sigma \in G$ \}. Com a ação de $G$ em $M$ dada por $\sigma . y=u_{\sigma} y$, para todo $y \in M$.

De fato, seja $M$ um $V$-módulo à esquerda, como $V$ é um $L$-espaço vetorial, obtemos que $M$ é também um $L$-espaço vetorial.

Sejam $\left\{x_{1}, \ldots, x_{n}\right\} \subset L$ uma base de $L$ sobre $K$ e $\phi_{1}, \ldots, \phi_{n} \in H_{o m}(L, K)$, tais que $\phi_{i}\left(x_{j}\right)=\delta_{i j}$, a base dual. Se $\phi \in \operatorname{Hom}_{K}(L, K)$, então $\phi$ pode ser estendida trivialmente a um $K$-endomorfismo de $L, \operatorname{logo} \operatorname{Hom}_{K}(L, K)$ pode ser visto como um subconjunto de $\operatorname{End}_{K}(L)$. Desde que $\Psi$ é um isomorfismo, podemos tomar $\nu_{i}=\Psi^{-1}\left(\phi_{i}\right) \in V$, para cada $i=1, \ldots, n$.

Mostraremos que a inversa de $\mu$ é a aplicação $\nu: M \rightarrow L \otimes_{K} M^{G}$, definida por $\nu(y)=\sum_{i=1}^{n} x_{i} \otimes \nu_{i} y$, para todo $y \in M$

Para mostrarmos que $\nu$ é a inversa de $\mu$, devemos mostrar inicialmente que

(a) $\nu_{i} y \in M^{G}$, para todo $1 \leq i \leq n$ e $y \in M$.

(b) $\sum_{i=1}^{n} x_{i} \nu_{i}=u_{1}$, onde $u_{1}=u_{\sigma}$, com $\sigma=i d \in G$.

(c) $\nu(x y)=(\Psi(\nu)(x)) y$, para todo $x \in L, \nu \in V$ e $y \in M^{G}$.

Para demonstrarmos (a), observemos que, para todo $x \in L$,

$$
\Psi\left(u_{\sigma} \nu_{i}\right)(x)=\Psi\left(u_{\sigma}\right)\left(\Psi\left(\nu_{i}\right)(x)\right)=\sigma\left(\phi_{i}(x)\right)=\phi_{i}(x)
$$

pois $\phi_{i}(x) \in K$. Logo $\Psi\left(u_{\sigma} \nu_{i}\right)=\phi_{i}=\Psi\left(\nu_{i}\right)$ e, como $\Psi$ é um isomorfismo, obtemos $u_{\sigma} \nu_{i}=\nu_{i}$ para todo $\sigma \in G$ e $1 \leq i \leq n$. Assim, para todo $y \in M$ e $1 \leq i \leq n$ temos que $\sigma\left(\nu_{i} y\right)=u_{\sigma} \nu_{i} y=\nu_{i} y$, para todo $\sigma \in G$, o que mostra que $\nu_{i} y \in M^{G}$. 
O item (b) segue da igualdade

$$
\Psi\left(\sum_{i=1}^{n} x_{i} \nu_{i}\right)(x)=\sum_{i=1}^{n} \Psi\left(\nu_{i}\right)(x)=\sum_{i=1}^{n} x_{i} \phi_{i}(x)=x=\Psi\left(u_{1}\right)(x),
$$

para todo $x \in L$ e do fato de $\Psi$ ser injetor.

Para mostrarmos (c), tomemos $x \in L, \nu=\sum_{\sigma \in G} a_{\sigma} u_{\sigma} \in V$ e $y \in M^{G}$. Então

$$
\begin{gathered}
\nu(x y)=\left(\nu\left(x u_{1}\right)\right) y=\left(\left(\sum_{\sigma \in G} a_{\sigma} u_{\sigma}\right)\left(x u_{1}\right)\right) y= \\
\left(\sum_{\sigma \in G} a_{\sigma} \sigma(x) u_{\sigma}\right) y=\left(\sum_{\sigma \in G} a_{\sigma} \sigma(x)\right) y=\Psi(\nu)(x) y .
\end{gathered}
$$

Mostraremos agora que $\nu$ é a inversa de $\mu$. Para cada $y \in M$, temos $\mu(\nu(y))=$ $\mu\left(\sum_{i=1}^{n} x_{i} \otimes \nu_{i}(y)\right)=\left(\sum_{i=1}^{n} x_{i} \nu_{i}\right)(y)=u_{1}(y)=y$. Para cada $x \in L$ e $y \in M^{G}$, temos $\nu(\mu(x \otimes y))=\nu(x y)=\sum_{i=1}^{n} x_{i} \otimes \nu_{i}(x y)=\sum_{i=1}^{n} x_{i} \otimes \Psi\left(\nu_{i}\right)(x)(y)=\sum_{i=1}^{n} x_{i} \otimes \phi_{i}(x)(y)=$ $\sum_{i=1}^{n} x_{i} \phi_{i}(x) \otimes y=x \otimes y$. Portanto $\mu$ é um isomorfismo de $L$-espaços vetoriais.

Seja $\varphi: L \otimes_{L} L \rightarrow L^{|G|}$, definida por $\varphi(x \otimes z)=(x \sigma(z))_{\sigma \in G}$. Mostremos que $\varphi$ é um isomorfismo de $L$-álgebras. Observe que

$$
L^{|G|}=L \times \cdots \times L \cong \operatorname{Map}(G, L)
$$

onde o isomorfismo é dado por

$$
\begin{aligned}
\left(a_{\sigma}\right)_{\sigma \in G} \leftrightarrow \phi: \quad G & \rightarrow L \\
\sigma & \mapsto a_{\sigma}
\end{aligned}
$$

Logo, $\operatorname{Map}(G, L)$ é uma $L$-álgebra e, $G$ age em $\operatorname{Map}(G, L)$ através da ação $\sigma . \phi=$ $\sigma \circ \phi \circ \sigma^{-1}$, para cada $\phi \in \operatorname{Map}(G, L)$ e para cada $\sigma \in G$. Essa ação induz uma estrutura de $V$-módulo à esquerda em $\operatorname{Map}(G, L)$.

Tomando $M=\operatorname{Map}(G, L)$, temos que $\mu: L \otimes_{K} \operatorname{Map}(G, L)^{G} \rightarrow \operatorname{Map}(G, L)$ é um isomorfismo de $L$-álgebras. Resta mostrarmos que $\operatorname{Map}(G, L)^{G} \cong L$. Para tanto, 
considere

$$
\begin{aligned}
\Lambda: \quad \operatorname{Map}(G, L)^{G} & \rightarrow \quad L \\
\phi & \mapsto \phi(1) .
\end{aligned}
$$

Claramente $\Lambda$ é um homomorfismo de $L$-álgebras. Seja

$$
\begin{array}{clcc}
\Lambda^{\prime}: & L & \rightarrow & \operatorname{Map}(G, L)^{G} \\
x & \mapsto & \phi_{x}
\end{array}
$$

onde $\phi_{x}(\sigma)=\sigma(x)$, para todo $x \in L$ e $\sigma \in G$.

Assim, para cada $x \in L$ temos $\Lambda \circ \Lambda^{\prime}(x)=\Lambda\left(\Lambda^{\prime}(x)\right)=\Lambda\left(\phi_{x}\right)=\phi_{x}(1)=1(x)=x$, e para cada $\phi \in \operatorname{Map}(G, L)^{G},\left(\Lambda^{\prime} \circ \Lambda\right)(\phi)=\Lambda^{\prime}(\phi(1))=\phi_{\phi(1)}$. Mas $\phi_{\phi(1)}(\sigma)=\sigma(\phi(1))=$ $\sigma\left(\phi\left(\sigma^{-1} \sigma\right)\right)=(\sigma . \phi)(\sigma)=\phi(\sigma)$, pois $\phi \in \operatorname{Map}(G, L)^{G}$. Logo $\left(\Lambda^{\prime} \circ \Lambda\right)(\phi)=\phi$ e portanto $\Lambda$ é um isomorfismo.

Compondo os isomorfismos

$$
L \otimes_{K} L \cong L \otimes_{K} \operatorname{Map}(G, L)^{G} \cong \operatorname{Map}(G, L) \cong L^{|G|},
$$

temos

$$
x \otimes z \mapsto x \otimes \phi_{z} \mapsto x \phi_{z} \mapsto\left(x \phi_{z}(\sigma)\right)_{\sigma \in G}=(x \sigma(z))_{\sigma \in G},
$$

que é o isomorfismo $\Phi$ desejado.

No restante desta seção, iremos introduzir a noção de forma traço e alguns resultados básicos, que são excenciais para o desenvolvimento do conteúdo do capítulo 2.

Sejam $L \supseteq K$ uma extensão galoisiana de corpos e $G=\operatorname{Gal}(L / K)=\left\{\sigma_{1}=\right.$ $\left.i d, \sigma_{2}, \ldots, \sigma_{n}\right\}$ o seu grupo de Galois. Para $x \in L$, o elemento, $\operatorname{Tr}_{L / K}(x)=\sum_{i=1}^{n} \sigma_{i}(x)$, é dito ser o traço de $L$ sobre $K$ de $x$.

Desde que $\sigma\left(\operatorname{Tr}_{L / K}(x)\right)=\operatorname{Tr}_{L / K}(x)$, para todo $\sigma \in G$ e $x \in L$, temos que $\operatorname{Tr}_{L / K}(x)$ pertence a $K$, ou seja, $\operatorname{Tr}_{L / K}$ é uma função de $L$ em $K$, claramente $K$-linear.

Agora, do fato de $\operatorname{Tr}_{L / K}$ ser $K$-linear e das propriedades dos elementos de $G$, é fácil ver que $T: L \times L \rightarrow K$, definida por $T(x, y)=\operatorname{Tr}_{L / K}(x y)=\sum_{\sigma \in G} \sigma(x y)$, para todo $x, y \in L$, é uma forma bilinear simétrica de $L$ sobre $K$ dita ser a forma traço de $L$ sobre $K$. 
A seguir, apresentamos um primeiro resultado sobre a forma traço, que será usado várias vezes no que segue.

Lema 1.14 Sejam $L \supseteq K$ uma extensão galoisiana com grupo de Galois $G$ e $T: L \times L \rightarrow K$ a forma traço de $L$ sobre $K$. Então a condição $T\left(\sigma_{i}(a), \sigma_{j}(a)\right)=\delta_{i j}$, para cada $\sigma_{i}, \sigma_{j} \in G$ e $a \in L$, é equivalente a

$$
T(a, \sigma(a))= \begin{cases}0, & \text { se } \sigma \neq i d \\ 1, & \text { se } \sigma=i d\end{cases}
$$

para todo $\sigma \in G$.

Demonstração: Como $T$ é $G$-invariante, para todo $a \in L$ e $\sigma_{i}, \sigma_{j} \in G$, temos que $T\left(\sigma_{i}(a), \sigma_{j}(a)\right)=T\left(a, \sigma_{i}^{-1} \sigma_{j}(a)\right)$. Assim $T\left(\sigma_{i}(a), \sigma_{j}(a)\right)=\delta_{i j}$ se, e somente se $\sigma_{i}=\sigma_{j}$ e, o resultado segue.

Nosso próximo objetivo é mostrarmos que se $L \supseteq K$ é uma extensão galoisiana de corpos, então a forma traço é não-singular, ou seja, a sua adjunta $\varphi: L \rightarrow L^{*}$, dada por $\varphi(x)(y)=T(x, y)$, para todo $x, y \in L$, é um isomorfismo de $K$-espaços vetoriais.

Teorema 1.15 Se $L \supseteq K$ é uma extensão galoisiana de corpos, então a forma traço $T$ de $L$ sobre $K$ é não-singular.

Demonstração: Basta mostrarmos que a matriz da adjunta de $T, \varphi$ com relação a um par de bases $B$ e $C$ de $L$ e $L^{*}$ sobre $K$, respectivamente, é inversível. Se $\mathcal{B}=\left\{e_{1}, e_{2}, \ldots, e_{n}\right\}$ é uma base de $L$ sobre $K$, escolhemos para $L^{*}$ a base dual $\mathcal{B}^{*}=\left\{f_{1}, \ldots, f_{n}\right\}$, onde $f_{i}\left(e_{j}\right)=\delta_{i j}$, para todo $i, j \in\{1, \ldots, n\}$. Com isso, temos que se $f \in L^{*}$, então $f$ se escreve de modo único, como

$$
f=\sum_{j=1}^{n} f\left(e_{j}\right) f_{j}
$$

Para cada $i=1, \cdots, n$, temos

$$
\varphi\left(e_{i}\right)=\sum_{j=1}^{n} \varphi\left(e_{i}\right)\left(e_{j}\right) f_{j}=\sum_{j=1}^{n} T\left(e_{i}, e_{j}\right) f_{j} .
$$


Logo, a matriz de $\varphi$ em relação às bases $\mathcal{B}$ e $\mathcal{B}^{*}$ é $\left(T\left(e_{i}, e_{j}\right)\right)_{1 \leq i, j \leq n}$. Queremos então mostrar que $\operatorname{det}\left(T\left(e_{i}, e_{j}\right)\right) \neq 0$.

Como $L \supseteq K$ é uma extensão finita e separável de corpos, então do corolário 1.4 temos que $L=K(a)$, para algum $a \in L$. Além disso, pelo primeiro teorema do isomorfismo, temos que $K(a) \cong \frac{K[X]}{[m(X)]}$, onde $m(X)$ é o polinômio minimal de $a$ sobre $K$. Do fato de $m(a)=0$, temos que $\sigma(m(a))=0$, ou seja, $m(\sigma(a))=0$, para cada $\sigma \in G=\operatorname{Gal}(L / K)$. Como a extensão $L \supseteq K$ é separável, temos que $\sigma(a), \operatorname{com} \sigma \in G$, são todas as raízes distintas de $m(X)$, e portanto $m(X)=\prod_{\sigma \in G}(X-\sigma(a))$.

Mais ainda, se $a=a_{1}, a_{2}, \ldots, a_{n}$ são as raízes de $m(X)$, temos do fato de $L \supseteq K$ ser separável, que são todas raízes distintas. Como podemos escolher $\mathcal{B}$ uma base de $L$ sobre $K$ arbitrária, tomamos $\mathcal{B}=\left\{1, a, \ldots, a^{n-1}\right\}$. Se $G=\left\{\sigma_{1}, \ldots, \sigma_{n}\right\}$ e $a_{j}=\sigma_{j}(a)$, para todo $1 \leq j \leq n$, temos que $a_{j}^{i}=\sigma_{j}\left(a^{i}\right)$. Assim,

$$
T\left(a^{i}, a^{j}\right)=\operatorname{Tr}_{L / K}\left(a^{i+j}\right)=\sum_{r=1}^{n} \sigma_{r}\left(a^{i+j}\right) .
$$

Logo, escrevendo $e_{i}=a^{i-1}$ para cada $i=1, \ldots, n$, temos

$$
\begin{aligned}
& \operatorname{det}\left(T\left(e_{i}, e_{j}\right)\right)=\operatorname{det}\left(T\left(a^{i-1}, a^{j-1}\right)\right)= \\
& \operatorname{det}\left[\begin{array}{cccc}
T(1,1) & T(1, a) & \cdots & T\left(1, a^{n-1}\right) \\
T(a, 1) & T(a, a) & \cdots & T\left(a, a^{n-1}\right) \\
\vdots & & & \vdots \\
T\left(a^{n-1}, 1\right) & \cdots & \cdots & T\left(a^{n-1}, a^{n-1}\right)
\end{array}\right]= \\
& \operatorname{det}\left[\begin{array}{ccc}
\operatorname{Tr}_{L / K}(1) & \cdots & \operatorname{Tr}_{L / K}\left(a^{n-1}\right) \\
\vdots & & \vdots \\
\operatorname{Tr}_{L / K}\left(a^{n-1}\right) & \cdots & \operatorname{Tr}_{L / K}\left(a^{2(n-1)}\right)
\end{array}\right]= \\
& \operatorname{det}\left[\begin{array}{ccc}
\sum_{r=1}^{n} 1 & \cdots & \sum_{r=1}^{n} a_{r}^{n-1} \\
\vdots & & \vdots \\
\sum_{r=1}^{n} a_{r}^{n-1} & \cdots & \sum_{r=1}^{1} a_{r}^{2(n-1)}
\end{array}\right]=
\end{aligned}
$$




$$
\begin{aligned}
& =\operatorname{det}\left(\left[\begin{array}{ccc}
1 & \cdots & 1 \\
a_{1} & \cdots & a_{n} \\
\vdots & & \vdots \\
a_{1}^{n-1} & \cdots & a_{n}^{n-1}
\end{array}\right] \cdot\left[\begin{array}{cccc}
1 & a_{1} & \cdots & a_{1}^{n-1} \\
\vdots & \vdots & & \vdots \\
1 & a_{n} & \cdots & a_{n}^{n-1}
\end{array}\right]\right)= \\
& =\left(\operatorname{det}\left[\begin{array}{cccc}
1 & 1 & \cdots & 1 \\
a_{1} & a_{2} & \cdots & a_{n} \\
a_{1}^{2} & a_{2}^{2} & \cdots & a_{n}^{2} \\
\vdots & \vdots & & \vdots \\
a_{1}^{n-1} & a_{2}^{n-1} & \cdots & a_{n}^{n-1}
\end{array}\right]\right)^{2}= \\
& =\left(\prod_{1 \leq k<\ell \leq n}\left(a_{k}-a_{\ell}\right)\right)^{2} \neq 0,
\end{aligned}
$$

pois $a_{k} \neq a_{l}$, para todo $1 \leq k<\ell \leq n$, o que mostra que a forma traço é não-singular.

O próximo resultado estabelece o comportamento da função traço de uma cadeia de corpos $L \supseteq F \supseteq K$.

Teorema 1.16 Se $L \supseteq F \supseteq K$ são extensões galoisianas de corpos, então

$$
\operatorname{Tr}_{L / K}=\operatorname{Tr}_{F / K} \circ \operatorname{Tr}_{L / F}
$$

Demonstração: Sejam $\operatorname{Gal}(F / K)=\left\{\rho_{1}, \cdots, \rho_{m}\right\}$ e $\operatorname{Gal}(L / F)=\left\{\phi_{1}, \cdots, \phi_{r}\right\}$. Por um resultado básico da teoria de corpos, podemos estender, de modo único, cada $\rho_{i}$, com $i=1, \cdots, m$, a um $K$-automorfismo $\overline{\rho_{i}}$ de $L$. Denotaremos $\overline{\rho_{i}}$ por $\rho_{i}$ para não carregar a notação. Para todo $i=1, \cdots, r$ e $j=1, \cdots, m$, temos que $\rho_{j} \phi_{i}$ é um $K$ automorfismo de $L$, e portanto $\rho_{j} \phi_{i} \in \operatorname{Gal}(L / K)$. Observe também que $|\operatorname{Gal}(L / K)|=$ $[L: K]=[L: F][F: K]=|\operatorname{Gal}(L / F)||\operatorname{Gal}(F / K)|=r m$ e portanto $\operatorname{Gal}(L / K)=$ $\left\{\rho_{j} \phi_{i} ; 1 \leq i \leq r\right.$ e $\left.1 \leq j \leq m\right\}$. Desta forma, $\operatorname{Tr}_{F / K} \circ \operatorname{Tr}_{L / F}(a)=\operatorname{Tr}_{F / K}\left(\sum_{i=1}^{r} \phi_{i}(a)\right)=$ $\sum_{j=1}^{m} \rho_{j}\left(\sum_{i=1}^{r} \phi_{i}(a)\right)=\sum_{j=1}^{m} \sum_{i=1}^{r} \rho_{j} \phi_{i}(a)=\operatorname{Tr}_{L / K}(a)$, para cada $a \in K$ 


\subsection{O teorema da base normal}

Esta seção tem por objetivo demonstrar a existência de bases normais para extensões galoisianas de corpos. Como o leitor pode notar esse resultado é o primeiro passo para garantir a existência das bases normais auto-duais no caso em que o grau da extensão é ímpar.

Sejam $L \supseteq K$ uma extensão sepáravel de corpos com $[L: K]=n$, e seja $F \supseteq K$ o fêcho normal de $L \supseteq K$.

Teorema 1.17 Com $F \supseteq L \supseteq K$ como acima, o número de imersões de $L$ em $F$ é $n=[L: K]$ e, se $\sigma_{1}=i d, \sigma_{2}, \ldots \sigma_{n}$ são tais imersões, então a sequência de $n$ elementos de $L\left\{a_{1}, a_{2}, \cdots, a_{n}\right\}$ é uma base de $L$ sobre $K$ se, e somente se

$$
\operatorname{det}\left(\begin{array}{cccc}
a_{1} & a_{2} & \cdots & a_{n} \\
\sigma_{2}\left(a_{1}\right) & \sigma_{2}\left(a_{2}\right) & \cdots & \sigma_{2}\left(a_{n}\right) \\
\vdots & \vdots & \vdots & \vdots \\
\sigma_{n}\left(a_{1}\right) & \sigma_{n}\left(a_{2}\right) & \cdots & \sigma_{n}\left(a_{n}\right)
\end{array}\right) \neq 0
$$

Demonstração: Note que $F \supseteq K$ é uma extensão galoisiana. Sejam $G=\operatorname{Gal}(F / K)$ e $H$ o subgrupo de $G$ formado pelos elementos que fixam os elementos de $L$. Então do teorema 1.2 (teorema fundamental de Galois), temos que $n=[L: K]=[G: H]$ e, podemos escrever

$$
G=\phi_{1} H \dot{\cup} \phi_{2} H \dot{\cup} \ldots \dot{\cup} \phi_{n} H
$$

onde $\phi_{i} H$, com $i=1, \ldots, n$, são as classes laterais distintas de $H$ em $G$ e $\phi_{1}=i d$.

Para cada $i=1, \cdots, n$ seja $\sigma_{i}=\left.\phi_{i}\right|_{L}$. Então $\sigma_{i}$ é uma imersão de $L$ em $F$ e, $\sigma_{i} \neq \sigma_{j}$, se $i \neq j$. De fato, se $\sigma_{i}=\sigma_{j}$, com $i \neq j$, então $\phi_{i}^{-1}\left(\phi_{j}(a)\right)=a$, para todo $a \in L$. Assim $\phi_{i}^{-1} \phi_{j} \in H$ e, conseqüentemente $\phi_{i} H=\phi_{j} H$, contrariando a hipótese.

Seja $\sigma$ uma imersão de $L$ em $F$. Como $F \supseteq K$ é normal e finita, temos que $F$ é corpo de raízes de um polinômio $f(X) \in K[X]$. Como $L \subseteq F$ e $\sigma(L) \subseteq F$, temos que $F$ é também corpo de raízes de $f(X)$ sobre $L$ e sobre $\sigma(L)$. Assim por um resultado conhecido de extensões de corpos, o isomorfismo $\sigma$ de $L$ em $\sigma(L)$ pode ser extendido 
a um automorfismo $\phi$ de $F$. Como $\phi \in \operatorname{Gal}(F / K)$, obtemos $\phi=\phi_{i} \lambda$, para algum $\lambda \in H$. Mas então $\sigma=\left.\phi\right|_{L}=\left.\phi_{i}\right|_{L}=\sigma_{i}$, para cada $i=1, \ldots, n$. Mostramos assim que $\sigma_{1}, \sigma_{2}, \ldots, \sigma_{n}$ formam a lista de todas as imersões de $L$ em $F$.

Suponhamos agora que os elementos $a_{1}, a_{2}, \ldots, a_{n}$ de $L$ são linearmente dependentes sobre $K$. Então existem $c_{i} \in K$, com $i=1, \ldots, n$, não todos nulos tais que

$$
c_{1} a_{1}+c_{2} a_{2}+\cdots+c_{n} a_{n}=0
$$

Aplicando $\sigma_{j}$ na igualdade acima, para cada $j=1, \ldots, n$, temos

$$
c_{1} \sigma_{j}\left(a_{1}\right)+\cdots+c_{n} \sigma_{j}\left(a_{n}\right)=0 .
$$

Com isso obtemos que o seguinte sistema linear homogêneo admite uma solução não trivial.

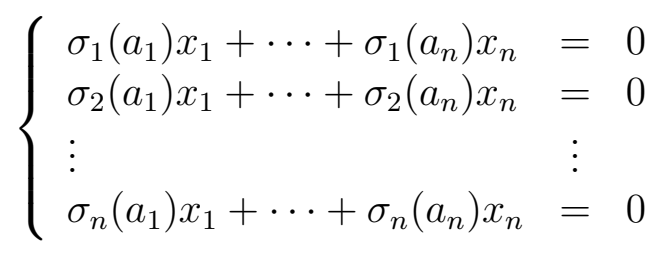

Conseqüentemente, temos que $\operatorname{det}\left(\sigma_{j}\left(a_{i}\right)\right) \neq 0$.

Reciprocamente, suponhamos que $\operatorname{det}\left(\sigma_{j}\left(a_{i}\right)\right)=0$. Então o sistema acima é indeterminado, ou seja, admite uma solução não trivial $\left(c_{1}, c_{2}, \ldots, c_{n}\right) \in K^{n}$. Neste caso, $\left\{a_{1}, a_{2}, \ldots, a_{n}\right\}$ não é uma base de $L$ sobre $K$ pois, caso contrário, todo elemento $a \in L$ poderia ser escrito como

$$
a=d_{1} a_{1}+d_{2} a_{2}+\cdots+d_{n} a_{n}
$$

$\operatorname{com} d_{i} \in K$, para $1 \leq i \leq n$. Assim,

$$
\sum_{j=1}^{n} c_{j} \sigma_{j}(a)=\sum_{1 \leq i, j \leq n} c_{j} d_{i} \sigma_{j}\left(a_{i}\right)=\sum_{i=1}^{n} d_{i}\left(\sum_{j=1}^{n} c_{j} \sigma_{j}\left(a_{i}\right)\right)=\sum_{j=1}^{n} d_{i} 0=0 .
$$

Mas isto contradiz a independência linear das imersões $\sigma_{1}, \ldots, \sigma_{n}$ de $L$ sobre $K$, o que mostra que $\left\{a_{1}, \cdots, a_{n}\right\}$ é uma base de $L$ sobre $K$.

O próximo resultado sobre polinômios nos será útil no que segue. 
Lema 1.18 Se $F$ é um corpo infinito e $f\left(X_{1}, \ldots, X_{r}\right)$ é um polinômio não nulo em $F\left[X_{1}, \ldots, X_{r}\right]$, então existem $a_{1}, \ldots, a_{r}$ em $F$ tais que $f\left(a_{1} \ldots, a_{r}\right) \neq 0$.

Demonstração: Faremos a demonstração por indução sobre $r$. Se $r=1$, sabemos que um polinômio $f(X)$ de grau $n$, tem no máximo $n$ raízes. Como $F$ é um corpo infinito, temos que existe $a \in F$ tal que $f(a) \neq 0$. Agora assumiremos que $r>1$ e o resultado vale para $r-1$. Podemos escrever

$$
f\left(X_{1}, \ldots, X_{r}\right)=b_{0}+b_{1} X_{r}+b_{2} X_{r}^{2}+\cdots+b_{n} X_{r}^{n}
$$

onde $b_{i} \in F\left[X_{1}, \ldots, X_{r-1}\right]$, com $b_{n}=b_{n}\left(X_{1}, \ldots, X_{r-1}\right) \neq 0$. Pela hipótese de indução, sabemos que existem $a_{1}, \ldots, a_{r-1} \in F$, tais que $b_{n}\left(a_{1}, \ldots, a_{r-1}\right) \neq 0$. Assim,

$$
\begin{aligned}
f\left(a_{1}, \ldots, a_{r-1}, X_{r}\right)= & b_{0}\left(a_{1}, \ldots, a_{r-1}\right)+b_{1}\left(a_{1}, \ldots, a_{r-1}\right) X_{r}+\ldots \\
& +b_{n}\left(a_{1}, \ldots, a_{r-1}\right) X_{r}^{n} \in F\left[X_{r}\right]
\end{aligned}
$$

é um polinômio não nulo de grau $n$. Agora, basta escolher $a_{r} \in F$ tal que $f\left(a_{1}, \ldots, a_{r}\right) \neq$ 0 e o resultado segue.

Para o próximo resultado, necessitaremos da noção de imersões algebricamente independentes.

Sejam $L \supseteq K$ uma extensão separável de corpos, $F \supseteq K$ seu fêcho normal e $\sigma_{1}, \cdots, \sigma_{n}$, imersões de $L$ em $F$. Dizemos que $\sigma_{1}, \cdots, \sigma_{n}$ são algebricamente independentes sobre $F$ se o único polinômio $f\left(X_{1}, \cdots, X_{n}\right) \in F\left[X_{1}, \cdots, X_{n}\right]$ satisfazendo $f\left(\sigma_{1}(a), \cdots, \sigma_{n}(a)\right)=0$, para todo $a \in L$, é o polinômio nulo. Com esta noção temos:

Teorema 1.19 Sejam $K$ um corpo infinito, $L \supseteq K$ uma extensão separável de corpos e $F \supseteq K$ o seu fêcho normal. Se $n=[L: K]$ e $\sigma_{1}, \sigma_{2}, \ldots, \sigma_{n}$ são as imersões distintas de $L$ em $F$, então estas imersões são algebricamente independentes sobre $F$.

Demonstração: Seja $f\left(X_{1}, \ldots, X_{n}\right) \in F\left[X_{1}, \ldots, X_{n}\right]$ satisfazendo $f\left(\sigma_{1}(a), \ldots, \sigma_{n}(a)\right)$ $=0$, para todo $a \in L$. Temos que mostrar que $f$ é o polinômio nulo. Seja $\left\{a_{1}, \ldots, a_{n}\right\}$ 
uma base de $L$ sobre $K$. Para quaisquer $c_{i} \in K$, com $1 \leq i \leq n$, temos

$$
\begin{aligned}
0 & =f\left(\sigma_{1}\left(\sum_{i=1}^{n} c_{i} a_{i}\right), \ldots, \sigma_{n}\left(\sum_{i=1}^{n} c_{i} a_{i}\right)\right) \\
& =f\left(\sum_{i=1}^{n} c_{i} \sigma_{1}\left(a_{i}\right), \ldots, \sum_{i=1}^{n} c_{i} \sigma_{n}\left(a_{i}\right)\right) .
\end{aligned}
$$

Consideremos o polinômio $g$ definido por

$$
g\left(X_{1}, \ldots, X_{n}\right)=f\left(\sum_{i=1}^{n} \sigma_{1}\left(a_{i}\right) X_{i}, \ldots, \sum_{i=1}^{n} \sigma_{n}\left(a_{i}\right) X_{i}\right) .
$$

Temos $g\left(c_{1}, \ldots, c_{n}\right)=0$ para quaisquer $c_{i} \in K$, com $1 \leq i \leq n$.

Seja $\left\{v_{1}, \ldots, v_{m}\right\}$ uma base de $F$ sobre $K$. Então podemos escrever

$$
g\left(X_{1}, \ldots, X_{n}\right)=\sum_{j=1}^{m} g_{j}\left(X_{1}, \ldots, X_{n}\right) v_{j}
$$

onde $g_{j}\left(X_{1}, \ldots, X_{n}\right) \in K\left[X_{1}, \ldots, X_{n}\right]$.

A condição $g\left(c_{1}, \ldots, c_{n}\right)=0$, implica que $g_{j}\left(c_{1}, \ldots, c_{n}\right)=0$ para cada $j=1, \ldots, m$. Como, para cada $i=1, \ldots, n$, o polinômio $g$ se anula para todo $c_{i} \in K$, com $1 \leq i \leq n$, e $K$ é infinito, concluímos, pelo lema 1.18 , que $g_{j}\left(X_{1}, \ldots, X_{n}\right)=0$ para cada $j=1, \ldots, m$. Conseqüentemente $g\left(X_{1}, \ldots, X_{n}\right)=0$.

Agora, do teorema 1.17, temos que $\operatorname{det}\left(\sigma_{j}\left(a_{i}\right)\right) \neq 0$. Assim a matriz $\left(\sigma_{j}\left(a_{i}\right)\right)$ possui inversa $\left(d_{i j}\right) \in M_{n}(F)$.

Como $g\left(X_{1}, \ldots, X_{n}\right)=f\left(\sum_{i=1}^{n} \sigma_{1}\left(a_{i}\right) X_{i}, \ldots, \sum_{i=1}^{n} \sigma_{n}\left(a_{i}\right) X_{i}\right)$, temos que

$$
g\left(\sum_{j, k} d_{1 j} \sigma_{j}\left(a_{k}\right) X_{k}, \ldots, \sum_{j, k} d_{n j} \sigma_{j}\left(a_{k}\right) X_{k}\right)=f\left(X_{1}, \ldots, X_{n}\right) .
$$

Mas como $g\left(X_{1}, \ldots, X_{n}\right)=0$, temos que $f\left(X_{1}, \ldots, X_{n}\right)=0$, provando assim a independência algébrica de $\sigma_{1}, \ldots, \sigma_{n}$ sobre $F$.

O próximo resultado nos mostra que o grupo de Galois de uma extensão finita de corpos finitos é cíclica. 
Proposição 1.20 Sejam $K$ um corpo finito com $q=p^{m}$ elementos e $L \supseteq K$ uma extensão de grau n. Então, Gal $(L / K)$ é cíclico gerado pelo K-automorfismo $\sigma: a \mapsto$ $a^{q}$, para cada $a \in L$.

Demonstração: Usando que $\operatorname{Car}(K)=p$, é fácil ver que $\sigma: L \rightarrow L$ é um homomorfismo de corpos. Se $\sigma(a)=0$, para algum $a \in L$, então $a^{q}=0$ no corpo $L$, o que implica que $a=0$, mostrando assim que $\sigma$ é injetor. O fato de $L$ ser finito implica que $\sigma$ é também sobrejetor. Logo $\sigma$ é um automorfismo de $L$. Agora, como $|K|=q$, temos que $a^{q}=a$ para todo $a \in K$, o que mostra que $\sigma \in \operatorname{Gal}(L / K)$.

Mostramos agora que a ordem de $\sigma$ é $n$. Como $L$ tem $q^{n}$ elementos, temos que $a^{q^{n}}=a$, para todo $a \in L$. Dessa forma, $\sigma^{n}=1$. Se $\sigma^{r}=1$ para algum $r<n$, temos $a^{q^{r}}=a$, para cada $a \in L$, o que contradiz o fato de que o polinômio $X^{q^{r}}-X$ de grau $q^{r}$ tem no máximo $q^{r}$ raízes distintas em $L$. Portanto, a ordem de $\sigma$ é $n$. Seja $F=L^{[\sigma]}$. Pelo teorema 1.2 (teorema fundamental da teoria de Galois) temos que $[L: F]=n$ e $\operatorname{Gal}(L / F)=[\sigma]$. Por outro lado, como $\sigma \in \operatorname{Gal}(L / K)$, temos que $K \subseteq F$. Logo $n=[L: K]=[L: F][F: K]=n[F: K]$, o que mostra que $F=K$ e, então, $L \supseteq K$ é galoisiana com $\operatorname{Gal}(L / K)=[\sigma]$, como queríamos.

Podemos agora demonstrar o principal resultado deste capítulo que é o teorema da base normal. Tal resultado garante apenas a existência de base normal.

Teorema 1.21 (Teorema da Base Normal) Toda extensão galoisiana de corpos admite uma base normal.

Demonstração: Seja $L \supseteq K$ uma extensão galoisiana de corpos. Dividiremos a demonstração em duas partes, uma considerando $L \supseteq K$ uma extensão cíclica, ou seja, $\operatorname{Gal}(L / K)$ um grupo cíclico e a outra considerando $K$ um corpo infinito. Estas duas condições englobam todos os casos pois se $K$ é finito, então $L$ será finito e portanto pela proposição $1.20, L \supseteq K$ será uma extensão cíclica. 
Primeiro assumiremos $L \supseteq K$ cíclica $\operatorname{com} \operatorname{Gal}(L / K)=[\sigma]=\left\{i d, \sigma, \ldots, \sigma^{n-1}\right\}$, onde $n=[L: K]$.

Observe que $L$ é um $K$-espaço vetorial de dimensão finita e $\sigma: L \rightarrow L$ é um operador $K$-linear. Além disso, pelo fato de $|[\sigma]|=[L: K]$, temos que o polinômio minimal de $\sigma$ sobre $K$ irá coincidir com o seu polinômio característico. Portanto, pelo teorema da decomposição racional (7.2 em [4]), existirá $a \in \dot{L}$, tal que $\left\{a, \sigma(a), \cdots, \sigma^{n-1}(a)\right\}$ é uma base naturalmente normal de $L$ sobre $K$.

Faremos agora o caso em que $K$ é um corpo infinito. Pelo teorema 1.19, os $K$ automorfismos $\sigma_{1}, \ldots, \sigma_{n}$ de $L$ são algebricamente independentes sobre $K$. Temos do teorema 1.17 também que se $a \in L$, então $\left\{\sigma_{1}(a), \ldots, \sigma_{n}(a)\right\}$ é base de $L$ sobre $K$ se, e somente se

$$
\operatorname{det}\left(\sigma_{i} \sigma_{j}(a)\right) \neq 0
$$

Como $\sigma_{1}, \ldots, \sigma_{n}$ são algebricamente independentes sobre $L$, temos que existe um $a \in L$ tal que $\operatorname{det}\left(\sigma_{i} \sigma_{j}(a)\right) \neq 0$, pois o determinante é um polinômio em $n^{2}$ variáveis. Então $\left\{\sigma_{1}(a), \sigma_{2}(a), \ldots, \sigma_{n}(a)\right\}$ é uma base normal de $L$ sobre $K$, como queríamos demonstrar. 


\section{CAPÍTULO 2}

\section{Sobre a existência de bases normais auto-duais}

O objetivo deste capítulo é mostrarmos que toda extensão galoisiana, de grau ímpar, de corpos, admite uma base normal auto-dual. Para tanto, neste capítulo, $L \supseteq K$ é uma extensão galoisiana de corpos, $G=\operatorname{Gal}(L / K)=\left\{\sigma_{1}, \ldots, \sigma_{n}\right\}, T: L \times L \rightarrow K$ é a forma traço de $L$ sobre $K$, e $K[G]$ é a $K$-álgebra de grupo de $G$.

Seja $\mathcal{B}=\left\{e_{1}, e_{2}, \ldots, e_{n}\right\}$ uma base de $L$ sobre $K$. Dizemos que $\mathcal{B}=\left\{e_{1}, e_{2}, \ldots, e_{n}\right\}$ é uma base auto-dual se ela for sua própria base dual com relação a forma bilinear traço, isto é, $T\left(e_{i}, e_{j}\right)=\delta_{i j}$, para todo $1 \leq i, j \leq n$.

Dizemos que uma base normal $\{\sigma(a) ; \sigma \in G\}$ de $L$ sobre $K$ é uma base normal auto-dual de $L$ sobre $K$ se $T\left(\sigma_{i}(a), \sigma_{j}(a)\right)=\delta_{i j}$, para todo $1 \leq i, j \leq n$.

Denotamos por $-: K[G] \rightarrow K[G]$ a involução $K$-linear dada por $\bar{\sigma}=\sigma^{-1}$, para todo $\sigma \in G$.

O corpo $L$ é um $K[G]$-módulo livre de posto 1 , com a multiplicação por escalar dada por

$$
\left(c_{1} \sigma_{1}+\cdots+c_{n} \sigma_{n}\right) \cdot x=c_{1} \sigma_{1}(x)+\cdots+c_{n} \sigma_{n}(x)
$$

para todo $x \in L$ e $c_{1}, \cdots, c_{n} \in K$.

Com relação a estrutura destes módulos, temos

Lema 2.1 Se $a \in \dot{L}$, então $\mathcal{B}=\left\{\sigma_{1}(a), \ldots, \sigma_{n}(a)\right\}$ é uma base normal de $L$ sobre $K$ se, e somente se $\{a\}$ é uma base de $L$ sobre $K[G]$. 
Demonstração: Dado $x \in L$, existem $c_{i} \in K, i=1, \cdots, n$, tais que

$$
x=c_{1} \sigma_{1}(a)+\cdots+c_{n} \sigma_{n}(a)=\left(c_{1} \sigma_{1}+\cdots+c_{n} \sigma_{n}\right) \cdot a,
$$

onde $c_{1} \sigma_{1}+\cdots+c_{n} \sigma_{n} \in K[G]$. Portanto, $\{a\}$ gera $L$ sobre $K[G]$.

Além disso, se $\left(c_{1} \sigma_{1}+\cdots+c_{n} \sigma_{n}\right) \cdot a=0$, então $c_{1} \sigma_{1}(a)+\cdots+c_{n} \sigma_{n}(a)=0$ e como $\mathcal{B}$ é linearmente independente sobre $K$, temos que $c_{1}=\cdots=c_{n}=0$ o que mostra que $\{a\}$ é linearmente independente sobre $K[G]$.

Por outro lado, se $\{a\}$ é uma base de $L$ sobre $K[G]$, para mostrarmos que $\mathcal{B}$ é uma base de $L$ sobre $K$, basta mostrarmos que $\mathcal{B}$ gera $L$, pois o número de elementos de $\mathcal{B}$ é $|G|=[L: K]$, pois $L \supseteq K$ é galoisiana. Dado $x \in L$, temos que existe $\sum_{i=1}^{n} c_{i} \sigma_{i} \in K[G]$ tal que

$$
x=\left(c_{1} \sigma_{1}+\cdots+c_{n} \sigma_{n}\right) \cdot a=c_{1} \sigma_{1}(a)+\cdots+c_{n} \sigma_{n}(a),
$$

o que mostra que $\mathcal{B}$ gera $L$ sobre $K$.

A forma bilinear traço $T: L \times L \rightarrow K$, induz uma aplicação $h_{T}: L \times L \rightarrow K[G]$ dada por

$$
h_{T}(x, y)=T\left(\sigma_{1}(x), y\right) \bar{\sigma}_{1}+\cdots+T\left(\sigma_{n}(x), y\right) \bar{\sigma}_{n}
$$

para todo $x, y \in L$.

A aplicação $h_{T}$ é uma forma hermitiana não-singular de $L$ sobre o anel $K[G]$. De fato, mostraremos inicialmente que $h_{T}$ é sesquilinear. Usando que $T$ é bilinear e $\sigma_{i} \in G$, obtemos facilmente que $h_{T}(x+y, z)=h_{T}(x, z)+h_{T}(y, z)$ e $h_{T}(x, y+z)=$ $h_{T}(x, y)+h_{T}(x, z)$, para todo $x, y, z \in L$.

Mais ainda, para todo $\sigma, \tau \in G$ e $x, y \in L$, temos:

$$
h_{T}(\sigma . x, \tau . y)=h_{T}(\sigma(x), \tau(y))=\sum_{i=1}^{n} T\left(\sigma_{i}(\sigma(x)), \tau(y)\right) \overline{\sigma_{i}} .
$$

Mas, do lema 1.14, temos $T\left(\sigma_{i}(\sigma(x)), \tau(y)\right)=T\left(\tau^{-1} \sigma_{i} \sigma(x), y\right)$. Logo,

$$
h_{T}(\sigma . x, \tau . y)=\sum_{i=1}^{n} T\left(\tau^{-1} \sigma_{i} \sigma(x), y\right) \overline{\sigma_{i}}=\sum_{i=1}^{n} T\left(\psi_{i}(x), y\right) \sigma \overline{\psi_{i}} \bar{\tau}=
$$




$$
\sigma\left(\sum_{i=1}^{n} T\left(\psi_{i}(x), y\right) \overline{\psi_{i}}\right) \bar{\tau}=\sigma h_{T}(x, y) \bar{\tau},
$$

de onde segue que $h_{T}(a . x, b . y)=a h_{T}(x, y) \bar{b}$, para todo $a, b \in K[G]$ e $x, y \in L$, mostrando assim que $h_{T}$ é sesquilinear.

Agora, para todo $x, y \in L$, temos

$$
\begin{gathered}
\overline{h_{T}(y, x)}=\overline{\sum_{i=1}^{n} T\left(\sigma_{i}(y), x\right) \overline{\sigma_{i}}}=\sum_{i=1}^{n} T\left(\sigma_{i}(y), x\right) \sigma_{i}= \\
\sum_{i=1}^{n} T\left(y, \sigma_{i}^{-1}(x)\right) \sigma_{i}=\sum_{i=1}^{n} T\left(\sigma_{i}^{-1}(x), y\right) \sigma_{i}=\sum_{i=1}^{n} T\left(\tau_{i}(x), y\right) \overline{\tau_{i}},
\end{gathered}
$$

onde $\tau_{i}=\sigma_{i}^{-1}$, para cada $i$, o que mostra que $\overline{h_{T}(y, x)}=h_{T}(x, y)$, ou seja, $h_{T}$ é uma forma hermitiana de $L$ sobre $K[G]$.

Mostraremos agora que $h_{T}$ é não-singular. Para tanto, note que do lema $2.1 L=$ $K[G] . a$, onde $\left\{\sigma_{1}(a), \cdots, \sigma_{n}(a)\right\}$ é uma base normal de $L$ sobre $K$. Assim, para mostrar que $h_{T}$ é não-singular, basta mostrar que $h_{T}(a, a) \neq 0$. Para isso, é suficiente mostrar que $T(\sigma(a), a) \neq 0$ para algum $\sigma \in G$. Mas se $T(\sigma(a), a)=0$, para todo $\sigma \in G$, e a matriz de $T$ em relação a base $\left\{\sigma_{1}(a), \cdots, \sigma_{n}(a)\right\}$ é

$$
\begin{gathered}
{\left[\begin{array}{cccc}
T(a, a) & T\left(a, \sigma_{2}(a)\right) & \ldots & T\left(a, \sigma_{n}(a)\right) \\
T\left(\sigma_{2}(a), a\right) & T\left(\sigma_{2}(a), \sigma_{2}(a)\right) & \ldots & T\left(\sigma_{2}(a), \sigma_{n}(a)\right) \\
\vdots & \vdots & \ddots & \vdots \\
T\left(\sigma_{n}(a), a\right) & T\left(\sigma_{n}(a), \sigma_{2}(a)\right) & \ldots & T\left(\sigma_{n}(a), \sigma_{n}(a)\right)
\end{array}\right]=} \\
{\left[\begin{array}{cccc}
0 & T\left(a, \sigma_{2}(a)\right) & \ldots & T\left(a, \sigma_{n}(a)\right) \\
0 & T\left(\sigma_{2}(a), \sigma_{2}(a)\right) & \ldots & T\left(\sigma_{2}(a), \sigma_{n}(a)\right) \\
\vdots & \vdots & \ddots & \vdots \\
0 & T\left(\sigma_{n}(a), \sigma_{2}(a)\right) & \ldots & T\left(\sigma_{n}(a), \sigma_{n}(a)\right)
\end{array}\right]}
\end{gathered}
$$

o que implicaria que $T$ seria singular, o que contradiz o teorema 1.15 , pois $L \supseteq K$ é separável.

O próximo resultado relaciona bases normais auto-duais de $L$ sobre $K$, com bases ortonormais de $L$ sobre $K[G]$. 
Lema 2.2 Se $a \in \dot{L}$, então $\mathcal{B}=\left\{\sigma_{1}(a), \ldots, \sigma_{n}(a)\right\}$ é uma base normal auto-dual de $L$ sobre $K$ se, e somente se $\{a\}$ é uma base ortonormal (relativamente a $h_{T}$ ) de $L$ sobre $K[G]$.

Demonstração: Se $\mathcal{B}$ é uma base normal auto-dual de $L$ sobre $K$, então $T\left(\sigma_{i}(a), \sigma_{j}(a)\right)$ $=\delta_{i j}$. Em particular, $T(\sigma(a), a)=0$, para $\sigma \neq i d$ e $T(\sigma(a), a)=1$ se $\sigma=i d$. Assim,

$$
h_{T}(a, a)=T\left(\sigma_{1}(a), a\right) \bar{\sigma}_{1}+\cdots+T\left(\sigma_{n}(a), a\right) \bar{\sigma}_{n}=i d=1 \in K[G] .
$$

Logo, $\{a\}$ é uma base ortonormal de $L$ sobre $K[G]$, com relação a $h_{T}$.

Por outro lado, se $h_{T}(a, a)=i d$, então

$$
T\left(\sigma_{1}(a), a\right) \bar{\sigma}_{1}+\cdots+T\left(\sigma_{n}(a), a\right) \bar{\sigma}_{n}=i d
$$

ou seja,

$$
\left(T\left(\sigma_{1}(a), a\right)-1\right) \cdot \bar{\sigma}_{1}+T\left(\sigma_{2}(a), a\right) \bar{\sigma}_{2}+\cdots+T\left(\sigma_{n}(a), a\right) \bar{\sigma}_{n}=0
$$

Como $G$ é uma base de $K[G]$ sobre $K$, obtemos que $T(\sigma(a), a)=i d$ se $\sigma=i d$ e $T(\sigma(a), a)=0$ se $\sigma \neq i d$. Agora, do lema 2.2 temos que a base normal $\mathcal{B}=$ $\left\{\sigma_{1}(a), \cdots, \sigma_{n}(a)\right\}$ é auto-dual.

Se $a \in \dot{L}$ gera uma base normal de $L$ sobre $K$, seja $u=h_{T}(a, a)$. Considerando as formas hermitianas de posto $1,\langle 1\rangle$ e $\langle u\rangle \simeq\left(L, h_{T}\right)$ sobre $K[G]$, temos:

Lema 2.3 A extensão galoisiana $L \supseteq K$ admite uma base normal auto-dual gerada por $a \in \dot{L}$ se, e somente se as formas hermitianas $\langle 1\rangle$ e $\langle u\rangle$ são isométricas sobre $K[G]$.

Demonstração: Se $a \in \dot{L}$ é um gerador da base normal auto-dual de $L$ sobre $K$, então do lema anterior, temos que $h_{T}(a, a)=1$. Portanto, a forma hermitiana $\langle u\rangle \simeq\langle 1\rangle$.

Por outro lado, se $\langle u\rangle \simeq\langle 1\rangle$, então $u=h_{T}(a, a)=1$ e, portanto do item (b) do lema anterior, temos que $\{a\}$ gera uma base normal auto-dual de $L$ sobre $K$. 
Lema 2.4 A álgebra de Galois $L \otimes_{K} L$ admite uma base normal auto-dual sobre $L$.

Demonstração: Pelo teorema 1.13 temos que se $L \supseteq K$ é galoisiana então $L^{|G|} \cong$ $L \otimes_{K} L$, como $K$-álgebras. Logo, basta mostrarmos que $L^{|G|}$ admite uma base normal auto-dual sobre $L$. Para cada $\sigma \in G$, definimos $e_{\sigma}=\left(x_{\rho}\right)_{\rho \in G} \in L^{|G|}$, onde $x_{\rho}=1$, se $\rho=\sigma$, e $x_{\rho}=0$, se $\rho \neq \sigma$.

Afirmamos que $\mathcal{B}=\left\{e_{\sigma}\right\}_{\sigma \in G}$ é uma base normal auto-dual de $L^{|G|}$ sobre $L$. De fato, em $L^{|G|}$ o produto é dado por $e_{\rho} e_{\sigma}=\delta_{\rho \sigma} \cdot e_{\rho \sigma}$ e note que $\sum_{\sigma \in G} e_{\sigma}=(1, \ldots, 1)$, ou seja, os elementos de $\mathcal{B}$ são idempotentes ortogonais de $L^{|G|}$, cuja soma é 1 em $L^{|G|}$.

Todo elemento de $L^{|G|}$ pode ser escrito na forma $\sum_{\sigma \in G} c_{\sigma} e_{\sigma}$, com $c_{\sigma} \in L$, e a ação de $G$ em $L^{|G|}$ é dada por

$$
\phi .\left(\sum_{\sigma \in G} c_{\sigma} e_{\sigma}\right)=\sum_{\sigma \in G} c_{\sigma} e_{\phi \sigma}
$$

para todo $\phi \in G$. Temos que $\mathcal{B}$ é uma base normal de $L^{|G|}$ sobre $L$, pois

$$
\left\{\sigma\left(e_{\rho}\right)\right\}_{\sigma \in G}=\left\{e_{\sigma \rho}\right\}_{\sigma \in G}=\left\{e_{\sigma}\right\}_{\sigma \in G}=\mathcal{B}
$$

Mostremos agora que essa base é auto-dual. Para todo $\sigma, \tau \in G$, temos

$$
T\left(e_{\sigma}, e_{\tau}\right)=\sum_{\rho \in G} \rho\left(e_{\sigma} e_{\tau}\right)
$$

Usando que $\sum_{\rho \in G} e_{\rho}=1$, temos que $\sum_{\rho \in G} \rho\left(e_{\sigma}\right)=1$. Portanto, $T\left(e_{\sigma}, e_{\tau}\right)=\delta_{\sigma \tau}$, ou seja, $\left\{e_{\sigma}\right\}_{\sigma \in G}$ é uma base normal auto-dual de $L^{|G|}$ sobre $L$.

Como conseqüência dos dois últimos lemas, temos

Corolário 2.5 As formas hermitianas $\langle 1\rangle$ e $\langle u\rangle$ são isométricas sobre $L[G]$.

Demonstração: No lema 2.4, mostramos que a álgebra de Galois $L \otimes_{K} L$ admite uma base normal auto-dual. Portanto, pelo lema 2.3, as formas hermitianas $\langle 1\rangle$ e $\langle u\rangle$ são isométricas sobre $L[G]$. 
Para os próximos dois resultados, assumiremos que $G=\operatorname{Gal}(L / K)=\left\{\sigma_{1}, \ldots, \sigma_{n}\right\}$, $\operatorname{Car}(K)=2, s=\sum_{i=1}^{n} \sigma_{i}, B=s K[G]$ é a subálgebra de $K[G]$ gerada por $s$ e $A=\frac{K[G]}{B}$ a álgebra quociente.

Observe que $s \sigma=\sigma s$, para cada $\sigma \in G$ e, conseqüentemente, $s x=x s$, para cada $x \in K[G]$. Logo, dado $b=s x \in B$, temos $\bar{b}=\overline{s x}=\overline{x s}=\overline{s x}=s \bar{x} \in B$, pois $\bar{s}=s$. Assim, $\bar{B}=B$ e a involução - de $K[G]$ induz uma involução - em $A$. Também assumiremos, no que segue, que $L \supseteq K$ é uma extensão de corpos de grau ímpar. Com as considerações acima, temos:

Lema 2.6 Temos que $K[G] \cong A \times K$, onde $A, K$ e $G$ são como acima.

Demonstração: Mostraremos inicialmente que $s$ é idempotente. De fato,

$$
s^{2}=\left(\sum_{i=1}^{n} \sigma_{i}\right)\left(\sum_{i=1}^{n} \sigma_{i}\right)=\sum_{i=1}^{n} \sigma_{1} \sigma_{i}+\sum_{i=1}^{n} \sigma_{2} \sigma_{i}+\cdots+\sum_{i=1}^{n} \sigma_{n} \sigma_{i}=n s=s,
$$

pois $n$ é ímpar e $\operatorname{Car}(K)=2$. Temos também $(1-s)^{2}=(1-s)$ e $s+(1-s)=1$. Observemos também que $s K[G]$ e $(1-s) K[G]$ possuem intersecção vazia. Mostraremos agora que

$$
K[G] \cong s K[G] \times(1-s) K[G] \cong B \times A .
$$

Consideremos $\varphi: K[G] \rightarrow s K[G] \times(1-s) K[G]$ definida por $\varphi(x)=(s x,(1-s) x)$, para todo $x \in K[G]$. Usando que $s$ e $(1-s)$ são idempotentes, obtemos facilmente que $\varphi$ é um homomorfismo de $K$-álgebras. Mais ainda, $\varphi$ é bijetora. De fato, se $\varphi(x)=0$, então $s x=0$ e $(1-s) x=0, \log 0(1-s) x=x-s x=x=0$ e, portanto, $\varphi$ é injetora. Seja $(s x,(1-s) y) \in s K[G] \times(1-s) K[G]$. Tomando $z=y-s y+s x \in K[G]$, temos que $\varphi(z)=(s x,(1-s) y)$. Logo, $\varphi$ é sobrejetora, e portanto, um isomorfismo de $K$-álgebras.

Mostremos agora que $A \cong(1-s) K[G]$, como $K$-álgebras. Para tanto, considere$\operatorname{mos} \varphi: K[G] \rightarrow(1-s) K[G]$, o homomorfismo sobrejetor de $K$-álgebras obtido pela composição de $\varphi$ a projeção na segunda coordenada, ou seja $\varphi(x)=(1-s) x$, para todo $x \in K[G]$. Note que $\varphi(x)=0$ se, e somente se $x=s x$ e, usando que $s$ é idem- 
potente, obtemos que o núcleo de $\varphi$ é $B$. Conseqüentemente, do primeiro teorema do isomorfismo, obtemos $A=\frac{K[G]}{B} \cong(1-s) K[G]$.

Resta apenas mostrarmos que $s K[G] \cong K$, como $K$-álgebras. Para isso, mostremos inicialmente que $s K[G]=s K$. De fato, para cada $x=c_{1} \sigma_{1}+\cdots+c_{n} \sigma_{n} \in K[G]$, temos

$$
\begin{aligned}
s x & =s\left(c_{1} \sigma_{1}+\cdots+c_{n} \sigma_{n}\right)=c_{1} s \sigma_{1}+\cdots+c_{n} s \sigma_{n}=c_{1} s+\cdots+c_{n} s \\
& =s\left(c_{1}+\cdots+c_{n}\right) \in s K
\end{aligned}
$$

e, claramente, $s K \subseteq s K[G]$. Portanto, $s K=s K[G]$. Por fim, basta notarmos que $s K \cong K$, pois a aplicação $\psi: s K \rightarrow K$, dada por $s a \mapsto a$, é um isomorfismo de $K$-álgebras. Portanto, $B=s K[G]=s K \cong K$ e assim, $K[G] \cong A \times K$, como queríamos.

Pelo lema 1.5 e pelo próximo lema, obtemos que todos os $A$-módulos hermitianos são pares.

Lema 2.7 Existe $a \in Z(A)$ que satisfazendo $a+\bar{a}=1$.

Demonstração: Como $|G|$ é ímpar, todo elemento de $G$ distinto da identidade não é conjugado do seu inverso.

Vamos supor inicialmente que a afirmação acima seja verdadeira. Em particular, temos que se $\sigma \in G$ e $\sigma \neq 1$, então $\sigma \neq \sigma^{-1}$ o que é imediato pelo teorema de Lagrange. Logo, existe um subconjunto $D$ de $G$ invariante por conjugação, isto é, $\sigma D \sigma^{-1}=D$, para todo $\sigma \in G$, tal que todo elemento distinto da identidade está em $D$ ou tem seu inverso em $D$, mas não em ambos, ou seja, $G=\{1\} \cup \dot{\cup} D^{-1}$, onde $D^{-1}=\left\{\sigma^{-1} \in G ; \sigma \in D\right\}$.

$$
\begin{aligned}
& \text { Tomemos } a=1+\sum_{\sigma \in D} \sigma . \text { Então } \\
& \qquad \begin{array}{l}
a+\bar{a}=1+\sum_{\sigma \in D} \sigma+1+\sum_{\sigma \in D} \sigma^{-1}=1+\sum_{\sigma \in G} \sigma=1+s=1,
\end{array}
\end{aligned}
$$

em $A=\frac{K[G]}{B}$ 
Mostremos agora que $a \in Z(A)$. Para isso, é suficiente mostrar que $a$ comuta com os elementos de $G$. De fato, para $\gamma \in G$, temos

$$
a \gamma=\left(1+\sum_{\sigma \in D} \sigma\right) \gamma=\gamma+\sum_{\sigma \in D} \sigma \gamma=\gamma+\sum_{\sigma \in D} \gamma \sigma=\gamma\left(1+\sum_{\sigma \in D} \sigma\right)=\gamma a
$$

onde usamos o fato que para cada $\sigma_{k} \in D$, existe $\sigma_{j} \in D$ tal que $\gamma \sigma_{k}=\sigma_{j} \gamma$, pois $D$ é invariante por conjugação.

Para terminarmos a demonstração resta apenas mostrarmos que a primeira afirmação é verdadeira.

Vamos supor por absurdo que exista $\gamma \in G$, distinto da identidade, tal que $\sigma \gamma \sigma^{-1}=$ $\gamma^{-1}$ para algum $\sigma \in G$. Seja $\bar{\gamma}$ a classe de conjugação de $\gamma$. Se $\varphi \in \bar{\gamma}$, então existe $\beta \in G$ tal que $\beta \gamma \beta^{-1}=\varphi$, $\log$ o $\varphi^{-1}=\beta^{-1} \gamma^{-1} \beta$, ou seja, $\varphi^{-1} \in \overline{\gamma^{-1}}$. Como $\overline{\gamma^{-1}}=\bar{\gamma}$, temos que $\varphi^{-1} \in \bar{\gamma}$. Como $|G|$ é ímpar, $\varphi \neq \varphi^{-1}$, para todo $\varphi \neq 1$ em $G$. Portanto, $\bar{\gamma}$ tem um número par de elementos, o que é um absurdo pois $|G|$ é ímpar e, a ordem de $G$ é divisível pelo número de elementos de suas classes de conjugação.

Possuímos agora todas as ferramentas que nos permitirão demonstrar o teorema principal deste capítulo.

Teorema 2.8 Se $L \supseteq K$ é uma extensão galoisiana de grau ímpar, então $L$ admite uma base normal auto-dual sobre $K$.

Demonstração: Se $\operatorname{Car}(K) \neq 2$, da observação 1.6, temos que toda forma hermitiana sobre corpos de característica distinta de 2 é par. Pelo corolário 2.5 e o teorema 1.10 podemos concluir que $\langle 1\rangle$ e $\langle u\rangle$ são isométricas sobre $K[G]$ e, do lema 2.3 teremos que $L$ possui uma base normal auto-dual sobre $K$.

Consideremos agora que $\operatorname{Car}(K)=2$. Sejam $s=\sum_{\sigma \in G} \sigma, B=s K[G]$ e $A=\frac{K[G]}{B}$. Vimos no lema 2.6 que $K[G] \cong A \times K$. Do lema 2.5, existe $y=\left(y_{A}, y_{K}\right) \in L[G] \cong A_{L} \times L$ tal que $y \bar{y}=u$ em $K[G]$, com $u=(v, z) \in A \times K$. Logo, $\left(y_{A}, y_{K}\right)\left(\overline{y_{A}}, \overline{y_{K}}\right)=(v, z)$, ou seja, $y_{A} \overline{y_{A}}=v$ e $y_{K} \overline{y_{K}}=z$. Como a involução em $K$ é a identidade, temos que 
$y_{K}^{2}=z \in K$, o que implica que $y_{K} \in K$. De fato, $y_{K}$ é raiz do polinômio $X^{2}-z \in K[X]$ e, portanto, $\left[K\left(y_{K}\right): K\right]=1$ ou 2. Como $\left[K\left(y_{K}\right): K\right]$ divide $[L: K]$ que é ímpar, temos $\left[K\left(y_{K}\right): K\right]=1$, isto é, $y_{K} \in K$. Pelos lemas 2.7 e 1.5, temos que toda forma hermitiana sobre $A$ é par.

Como $y_{A} \overline{y_{A}}=v$, temos que $\langle 1\rangle$ e $\langle v\rangle$ são isométricos sobre $A_{L}$. Do teorema 1.10, temos que $\langle 1\rangle$ e $\langle v\rangle$ são isométricos sobre $A$, isto é, existe $x_{A} \in A$ tal que $x_{A} \overline{x_{A}}=v$. Consideremos $x=\left(x_{A}, y_{K}\right) \in A \times K \cong K[G]$. Então, $x \bar{x}=\left(x_{A} \overline{x_{A}}, y_{K} \overline{y_{K}}\right)=(v, z)=u$ em $K[G]$. Portanto, $\langle 1\rangle$ e $\langle u\rangle$ são isométricas sobre $K[G]$ e do lema 2.3, temos que $L \supseteq K$ admite uma base normal auto-dual.

Finalizamos este capítulo com um exemplo:

Exemplo 2.9 Sejam $K=\mathbb{Q}$ e $f(X)=X^{3}-3 X+1 \in \mathbb{Q}[X]$. Então $L=\mathbb{Q}(a)$ com $a=X+(f(X))$ é uma extensão galoisiana de grau 3 e $G a l(L / K)=[\sigma]$, onde $\sigma$ é o $K$-automorfismo de $L$ é dado por $\sigma(a)=a^{2}-2$.

Seja $\alpha=-1+\frac{a}{3}+\frac{a^{2}}{3} \in L$. Afirmamos que $\mathcal{B}=\left\{\alpha, \sigma(\alpha), \sigma^{2}(\alpha)\right\}$ é uma base normal auto-dual de $L$ sobre $K$.

De fato, em $L$ temos

$$
\begin{aligned}
& \sigma(\alpha)=\left(\frac{a^{2}}{3}+\frac{a}{3}-1\right)^{2}-2=\frac{a^{4}}{9}+\frac{2 a^{3}}{9}-\frac{5 a^{2}}{9}-\frac{2 a}{3}-1=-\frac{2 a^{2}}{9}-\frac{a}{9}-\frac{11}{9}, \mathrm{e} \\
& \sigma^{2}(\alpha)=\left(\frac{a^{2}}{3}+\frac{a}{3}-1\right)^{4}-4\left(\frac{a^{2}}{3}+\frac{a}{3}-1\right)^{2}+2=\frac{19 a^{2}}{9}+\frac{10 a}{27}-\frac{5}{9} .
\end{aligned}
$$

Como $[L: K]=3$, basta mostrarmos que $\mathcal{B}$ é linearmente independente para ser uma base de $L$ sobre $K$.

Se $c_{1} \alpha+c_{2} \sigma(\alpha)+c_{3} \sigma^{3}(\alpha)=0, \operatorname{com} c_{i} \in K$, temos

$$
c_{1}\left(\frac{a^{2}}{3}+\frac{a}{3}-1\right)+c_{2}\left(-\frac{2 a^{2}}{9}-\frac{a}{9}-\frac{11}{9}\right)+c_{3}\left(\frac{19 a^{2}}{9}+\frac{10 a}{27}-\frac{5}{9}\right)=0
$$

Usando que $\left\{1, a, a^{2}\right\}$ é uma base de $L$ sobre $K$, obtemos que $c_{1}, c_{2}$ e $c_{3}$ satisfazem 
o seguinte sistema linear homogêneo:

$$
\begin{array}{r}
3 c_{1}-2 c_{2}+19 c_{3}=0 \\
9 c_{1}-3 c_{2}+10 c_{3}=0 \\
-9 c_{1}-11 c_{2}-5 c_{3}=0
\end{array}
$$

cuja única solução é a trivial. Com isso, temos que $\mathcal{B}$ é uma base normal de $\mathbb{Q}(a)$ sobre $\mathbb{Q}$.

Mostremos que $\mathcal{B}$ é auto-dual. Lembremos que, neste caso, a forma traço de $\mathbb{Q}(a)$ sobre $\mathbb{Q}$ é dada por $T(x, y)=x y+\sigma(x y)+\sigma^{2}(x y)$, para todo $x, y \in \mathbb{Q}(a)$. Com simples cálculos mostra-se que $T(\alpha, \alpha)=T(\sigma(\alpha), \sigma(\alpha))=T\left(\sigma^{2}(\alpha), \sigma^{2}(\alpha)\right)=\alpha^{2}+\sigma\left(\alpha^{2}\right)+$ $\sigma^{2}\left(\alpha^{2}\right) ; T(\alpha, \sigma(\alpha))=T\left(\alpha, \sigma^{2}(\alpha)\right)=T\left(\sigma(\alpha), \sigma^{2}(\alpha)\right)=\alpha \sigma(\alpha)+\alpha \sigma^{2}(\alpha)+\sigma(\alpha) \sigma^{2}(\alpha)$. Agora,

$$
\begin{aligned}
& \alpha^{2}=\frac{x^{4}}{9}+\frac{2 x^{3}}{9}-\frac{5 x^{2}}{9}-\frac{2 x}{3}+1 \\
& (\sigma(\alpha))^{2}=\sigma\left(\alpha^{2}\right)=\frac{4 x^{4}}{81}+\frac{4 x^{3}}{81}+\frac{45 x^{2}}{81}+\frac{22 x}{81}+\frac{121}{81}, \\
& \left(\sigma^{2}(\alpha)\right)^{2}=\sigma^{2}\left(\alpha^{2}\right)=\frac{361 x^{4}}{81}+\frac{380 x^{3}}{243}-\frac{1610 x^{2}}{729}-\frac{100 x}{243}+\frac{25}{81} .
\end{aligned}
$$

e, conseqüentemente,

$$
\begin{aligned}
\alpha^{2}+\sigma\left(\alpha^{2}\right)+\sigma^{2}\left(\alpha^{2}\right) & =\frac{374 X^{4}}{81}+\frac{227 X^{3}}{81}-\frac{374 X^{2}}{27}-\frac{307 X}{81}+\frac{308}{81} \\
& =\frac{374 X^{4}}{81}+\frac{227 X^{3}}{81}-\frac{374 X^{2}}{27}-\frac{307 X}{81}+\frac{227}{81}+\frac{81}{81} \\
& =\left(X^{3}-3 X+1\right)\left(\frac{374 X}{81}+\frac{227}{81}\right)+1 \\
& =1 .
\end{aligned}
$$

Mais ainda,

$$
\begin{aligned}
\alpha \sigma(\alpha)+\alpha \sigma^{2}(\alpha)+\sigma(\alpha) \sigma^{2}(\alpha) & =\frac{13 X^{4}}{81}-\frac{19 X^{3}}{81}-\frac{780 X^{2}}{243}-\frac{302 X}{243}+\frac{199}{81} \\
& =\left(\alpha^{3}-3 X+1\right)\left(\frac{13 X}{81}+\frac{199}{81}\right)=0
\end{aligned}
$$

o que mostra que $\mathcal{B}$ é uma base normal auto-dual de $\mathbb{Q}(a)$ sobre $\mathbb{Q}$. 


\section{CAPÍTULO 3}

\section{Sobre a não existência de bases normais auto-duais}

O principal resultado do capítulo 2 diz que toda extensão galoisiana de corpos de grau ímpar admite uma base normal auto-dual. A questão natural que surge é: As extensões galoisianas de corpos de grau par não admitem bases normais auto-duais? Neste capítulo, responderemos em parte esta pergunta, mostrando que em alguns tipos de extensões galoisianas de corpos de grau par não admitem bases normais ortogonais e conseqüentemente normais auto-duais.

O principal resultado deste capítulo é o teorema abaixo, que foi demonstrado por D. S. Kang em [6], a qual seguimos juntamente com [7], para fazermos o estudo que apresentaremos a seguir.

Teorema 3.1 Seja $G$ um grupo finito de ordem par.

(a) Se $G$ tem um subgrupo de indice 2 , então $L \supseteq K$ não admite base normal ortogonal para qualquer extensão de corpos $L \supseteq K$ com grupo de Galois $G$.

(b) Se um 2-subgrupo de Sylow de $G$ é abeliano, então $L \supseteq K$ não admite uma base normal ortogonal para alguma extensão de corpos $L \supseteq K$ com grupo de Galois $G$.

O restante do capítulo é dedicado a apresentação da demonstração deste teorema. Para tanto, apresentaremos algumas noções e resultados auxiliares. No que segue, os 
corpos considerados serão de característica distinta de 2 .

Sejam $L \supseteq K$ uma extensão galoisiana de corpos com grupo de Galois $G=$ $\operatorname{Gal}(L / K)$ e $T: L \times L \rightarrow K$ a forma bilinear traço de $L$ sobre $K$. Associada a esta forma bilinear, temos a forma quadrática, que também chamaremos de forma traço de $L$ sobre $K$, dada por $q_{L / K}(x)=T(x, x)=\operatorname{Tr}_{L / K}\left(x^{2}\right)$, para todo $x \in L$.

Para $a \in L, a \neq 0$, a forma quadrática $q_{L / K}^{a}$, definida por $q_{L / K}^{a}(x)=\operatorname{Tr}_{L / K}\left(a x^{2}\right)$, para todo $x \in L$, é dita ser a forma traço escalar de $L$ sobre $K$. Note que $q_{L / K}^{1}=q_{L / K}$.

O próximo lema determina a forma traço de uma extensão quadrática.

Lema 3.2 Seja $L=K(\sqrt{b}) \supseteq K$, para algum $b \in K$, uma extensão quadrática de corpos. Então $q_{L / K}=\langle 2,2 b\rangle$.

Demonstração: Neste caso, temos que $|G|=|\operatorname{Gal}(L / K)|=2$ e, portanto, $G=\{1, \sigma\}$, onde $\sigma(\sqrt{b})=-\sqrt{b}$. Vamos mostrar que, em relação a base $\{1, \sqrt{b}\}$ de $L$ sobre $K$, a forma quadrática $q_{L / K}$ tem a diagonalização $\langle 2,2 b\rangle$. De fato,

$$
\begin{aligned}
& q_{L / K}(1)=T(1,1)=1+\sigma(1)=2 ; \\
& T(1, \sqrt{b})=T(\sqrt{b}, 1)=\sqrt{b}+\sigma(\sqrt{b})=0 ; \\
& q_{L / K}(\sqrt{b})=T(\sqrt{b}, \sqrt{b})=b+\rho(\sqrt{b} \cdot \sqrt{b})=2 b,
\end{aligned}
$$

o que mostra que $q_{L / K}=\langle 2,2 b\rangle$.

Vejamos agora, como é o comportamento da forma traço com relação à uma cadeia de corpos.

Lema 3.3 Sejam $L \supseteq F \supseteq K$ extensões de corpos. Se $q_{L / F}=\left\langle a_{1}, a_{2}, \ldots, a_{n}\right\rangle$, então

(a) $q_{L / K}=q_{F / K}^{a_{1}} \perp q_{F / K}^{a_{2}} \perp \cdots \perp q_{F / K}^{a_{n}}$;

(b) Se $a_{i} \in K$, para todo $i=1, \ldots, n$, então $q_{L / K}=\left\langle a_{1}, a_{2}, \ldots, a_{n}\right\rangle \otimes q_{F / K}$.

Demonstração: (a) Seja $\left\{v_{1}, \ldots, v_{n}\right\}$ uma base de $L$ sobre $F$ tal que a decomposição de $q_{L / F}$, com relação a esta base, é $q_{L / F}=\left\langle a_{1}, a_{2}, \ldots, a_{n}\right\rangle$, com $a_{1}, \ldots, a_{n} \in \dot{F}$. Então, 
$L=F v_{1} \oplus F v_{2} \oplus \cdots \oplus F v_{n}$ como espaço vetorial sobre $K$. Mais ainda, esta soma é ortogonal com respeito a $q_{L / K}$, pois se $i \neq j$ e $c_{i}, c_{j} \in F$, então $T\left(c_{i} v_{i}, c_{j} v_{j}\right)=$ $\operatorname{Tr}_{L / K}\left(c_{i} c_{j} v_{i} v_{j}\right)=\operatorname{Tr}_{F / K} \circ \operatorname{Tr}_{L / F}\left(c_{i} c_{j} v_{i} v_{j}\right)$ do teorema 1.16. Como $T_{L / F}$ é $F$-linear, temos $T\left(c_{i} v_{i}, c_{j} v_{j}\right)=\operatorname{Tr}_{F / K}\left(c_{i} c_{j} T_{L / F}\left(v_{i} v_{j}\right)\right)=0$, pois $v_{i}$ e $v_{j}$ são vetores de uma base ortogonal de $L$ sobre $F$.

Agora, mostraremos que a restrição da forma traço $q_{L / K}$ a cada subespaço $F v_{i}$ é da forma, $q_{F / K}^{a_{i}}$, o que finaliza a demonstração do item (a).

Para cada $i=1, \cdots, n$, se $c \in F$, novamente usando o teorema 1.16 , temos

$$
\begin{gathered}
q_{L / K}\left(c v_{i}\right)=\operatorname{Tr}_{L / K}\left(c^{2} v_{i}^{2}\right)=\operatorname{Tr}_{L / K}\left(c^{2}\right) \operatorname{Tr}_{L / K}\left(v_{i}^{2}\right)= \\
\left(\operatorname{Tr}_{F / K} \circ \operatorname{Tr}_{L / F}\left(c^{2}\right)\right)\left(\operatorname{Tr}_{F / K} \circ \operatorname{Tr}_{L / F}\left(v_{i}^{2}\right)\right)= \\
\operatorname{Tr}_{F / K}\left(c^{2}\right) \operatorname{Tr}_{F / K}\left(a_{i}\right)=\operatorname{Tr}_{F / K}\left(a_{i} c^{2}\right)=q_{F / K}^{a_{i}}(c),
\end{gathered}
$$

como queríamos.

(b) Se $a_{i} \in K$, para todo $i=1, \ldots, n$, então, para cada $a \in F$, temos

$$
q_{F / K}^{a_{i}}(a)=\operatorname{Tr}_{F / K}\left(a_{i} a^{2}\right)=a_{i} \operatorname{Tr}_{F / K}\left(a^{2}\right)=\left\langle a_{i}\right\rangle \otimes q_{F / K}(1 \otimes a) .
$$

Assim, do item anterior, obtemos que

$$
\begin{aligned}
q_{L / K} & =q_{F / K}^{a_{1}} \perp q_{F / K}^{a_{2}} \perp \cdots \perp q_{F / K}^{a_{n}} \\
& =\left(\left\langle a_{1}\right\rangle \otimes q_{F / K}\right) \perp\left(\left\langle a_{2}\right\rangle \otimes q_{F / K}\right) \perp \cdots \perp\left(\left\langle a_{n}\right\rangle \otimes q_{F / K}\right) \\
& =\left\langle a_{1}, a_{2}, \ldots, a_{n}\right\rangle \otimes q_{F / K},
\end{aligned}
$$

como queríamos.

Seja $K$ um corpo contendo uma raiz $n$-ésima primitiva da unidade, para um inteiro positivo par $n$. Seja $L=K(\sqrt[n]{a})$, com $a \in \dot{K}$, uma extensão cíclica de $K$ de grau $n$, ou seja, cujo grupo de Galois $\operatorname{Gal}(L / K)$ é cíclico. Neste caso, temos que $\operatorname{Gal}(L / K)=[\sigma]$, onde $\sigma$ é o $K$-automorfismo de $L$ que leva $x=\sqrt[n]{a}$ em $\omega x$, com $\omega \in K$ uma raiz $n$-ésima 
primitiva da unidade. Mais ainda, para cada $i=1, \cdots, n, \sigma^{i}(x)=\omega^{i} x$. Com estas considerações, apresentamos a classe de $q_{L / K}$ no anel de Witt dos espaços bilineares sobre $K, W(K)$.

Lema 3.4 Para $L \supseteq K$ como acima, temos que $q_{L / K}=\langle n, n a\rangle$ em $W(K)$.

Demonstração: Considere a base de $L$ sobre $K,\left\{1, x, x^{2}, \cdots, x^{n-1}\right\}$, onde $x=\sqrt[n]{a}$. Como $n$ é par, podemos considerar o subespaço de $L$ gerado por $\left\{1, x^{n / 2}\right\}$.

Afirmamos que a forma traço $q_{L / K}$ restrita a este subespaço é $\langle n, n a\rangle$.

De fato, $q_{L / K}(1)=\operatorname{Tr}_{L / K}\left(1^{2}\right)=\sum_{i=1}^{n} \sigma^{i}(1)=|G|=n, q_{L / K}\left(x^{n / 2}\right)=\operatorname{Tr}_{L / K}\left(x^{n}\right)=$ $\operatorname{Tr}_{L / K}(a)=\sum_{i=1}^{n} \sigma^{i}(a)=\sum_{i=1}^{n} a \sigma^{i}(1)=a|G|=n a$, e $T\left(1, x^{n / 2}\right)=\operatorname{Tr}_{L / K}\left(x^{n / 2}\right)=$ $\sum_{i=1}^{n} \sigma^{i}\left(x^{n / 2}\right)$

Como $\sigma(x)=\omega x$, temos que $\sigma\left(x^{n / 2}\right)=\omega^{n / 2} x^{n / 2}$. Mas $\omega$ é raiz $n$-ésima primitiva da unidade e $n$ é par, então $\omega^{n / 2}=-1$, pois $\left(\omega^{n / 2}-1\right)\left(\omega^{n / 2}+1\right)=\omega^{n}-1=0$ e $\omega^{n / 2} \neq 1$. Assim, $\sigma\left(x^{n / 2}\right)=-x^{n / 2}$ o que implica que $\sigma^{i}\left(x^{n / 2}\right)=(-1)^{i} x^{n / 2}$, para cada $i=1, \cdots, n$. Logo $T\left(1, x^{n / 2}\right)=\sum_{i=1}^{n} \sigma^{i}\left(x^{n / 2}\right)=\sum_{i=1}^{n}(-1)^{i} x^{n / 2}=0$, pois $n$ é par, o que mostra a afirmação.

Agora, vamos mostrar que para $i=1, \cdots, \frac{n}{2}-1$, a forma traço $q_{L / K}$ restrita ao subespaço $K x^{i} \oplus K x^{n-i}$ é hiperbólica. Como $q_{L / K}\left(x^{i}\right)=\operatorname{Tr}_{L / K}\left(x^{2 i}\right)=\sum_{j=1}^{n} \sigma^{j}\left(x^{2 i}\right)$ e, $\sigma\left(x^{2 i}\right)=\omega^{2 i} x^{2 i}$, temos que $\sigma^{j}\left(x^{2 i}\right)=\omega^{2 i j} x^{2 i}$, de onde segue que

$q_{L / K}\left(x^{i}\right)=x^{2 i}\left(\omega^{2 i}+\left(\omega^{2 i}\right)^{2}+\cdots+\left(\omega^{2 i}\right)^{n-1}+\left(\omega^{2 i}\right)^{n}\right)=x^{2 i}\left(1+\omega^{2 i}+\left(\omega^{2 i}\right)^{2}+\cdots+\left(\omega^{2 i}\right)^{n-1}\right)$.

Mas, $2 i<n$, para cada $i=1, \ldots, \frac{n}{2}-1$, o que implica que $\omega^{2 i} \neq 1$ e, como $\omega^{2 i}$ é raiz do polinômio $f(X)=X^{n-1}+X^{n-2}+\cdots+1$, obtemos que $q_{L / K}\left(x^{i}\right)=0$, ou seja, $x^{i}$ é um vetor isotrópico e, como $T\left(x^{i}, x^{n-i}\right)=\operatorname{Tr}_{L / K}\left(x^{n}\right)=\operatorname{Tr}_{L / K}(a)=n a$, temos que $q_{L / K}$ restrito ao subespaço $K x^{i} \oplus K x^{n-i}$ é uma forma quadrática não-singular isotrópica. Conseqüentemente, do teorema 4.5 de [11], temos que esta restrição é isométrica ao plano hiperbólico $\langle 1,-1\rangle$. 
Assim, $q_{L / K} \simeq\langle n, n a\rangle \perp\left(\frac{n-2}{2}\right)\langle 1,-1\rangle$, o que mostra o lema.

Lema 3.5 Seja $L \supseteq K$ uma extensão galoisiana de corpos, com $q_{L / K}=\langle a, a\rangle$ para algum $a \in \dot{K}$. Então $q_{L / K}$ é hiperbólica se, e somente se $K$ contém uma raiz quarta da unidade.

Demonstração: Desde que uma forma quadrática de dimensão 2 é hiperbólica se, e somente se o seu determinante é menos um quadrado, temos que $q_{L / K}=\langle a, a\rangle$ é hiperbólica se, e somente se $a^{2}=\operatorname{det}\left(q_{L / K}\right)=-1 \mathrm{em} \dot{K} / \dot{K}^{2}$. Mas $a^{2}=-1 \mathrm{em} \dot{K} / \dot{K}^{2}$ se, e somente se $K$ contém uma raiz quarta primitiva da unidade, o que mostra o lema.

Nosso próximo objetivo é relacionarmos a existência de base normal ortogonal com o fato da forma traço $q_{L / K}$ ser hiperbólica. Para isso, se $L \supseteq K$ é uma extensão galoisiana de corpos com grupo de Galois $G$, dizemos que uma base normal $\{\sigma(a) ; \sigma \in G\}$ de $L$ sobre $K$ é uma base normal ortogonal se ela for ortogonal em relação a forma traço, ou seja,

$$
T\left(\sigma_{i}(a), \sigma_{j}(a)\right)=\operatorname{Tr}_{L / K}\left(\sigma_{i}(a) \sigma_{j}(a)\right)=0
$$

para todo $\sigma_{i} \neq \sigma_{j}$ em $G$.

Teorema 3.6 Sejam K um corpo contendo uma raiz quarta primitiva da unidade, $L \supseteq K$ uma extensão galoisiana com $G=\operatorname{Gal}(L / K)$ de ordem par. Se $L \supseteq K$ admite uma base normal ortogonal, então $q_{L / K}$ é hiperbólica.

Demonstração: Sejam $\mathcal{B}=\left\{\sigma_{1}(a), \sigma_{2}(a), \ldots, \sigma_{n}(a)\right\}$ uma base normal ortogonal de $L$ sobre $K$, onde $a \in \dot{L}$ e $n=[L: K]$ é par. Como a forma traço é uma aplicação $G$-invariante, isto é, $T(x, y)=T(\sigma(x), \sigma(y))$, para todo $x, y \in L$ e $\sigma \in G$, temos que existe $c=\operatorname{Tr}_{L / K}\left(a^{2}\right) \in \dot{K}$ tal que

$$
T(\sigma(a), \sigma(a))=\operatorname{Tr}_{L / K}(\sigma(a) \sigma(a))=\operatorname{Tr}_{L / K}\left(\sigma\left(a^{2}\right)\right)=\operatorname{Tr}_{L / K}\left(a^{2}\right)=c
$$


para cada $\sigma \in G$.

Assim, a decomposição de $q_{L / K}$ com relação a base $\mathcal{B}$ é $q_{L / K}=\langle c, c, \cdots, c\rangle=$ $\langle c, c\rangle \perp\langle c, c\rangle \perp \cdots \perp\langle c, c\rangle=\frac{n}{2}\langle c, c\rangle$.

Como $K$ contém uma raiz quarta primitiva da unidade, teremos pelo lema anterior que a forma $\langle c, c\rangle$ é hiperbólica e, conseqüentemente, $q_{L / K}$ é hiperbólica.

Como conseqüência imediata temos

Corolário 3.7 Seja $L \supseteq K$ uma extensão galoisiana de corpos. Se L sobre $K$ admitir uma base normal ortogonal, então $\operatorname{det}\left(q_{L / K}\right)$ é um quadrado em $\dot{K}$.

Demonstração: Como a forma traço é uma aplicação $G$-invariante, como na demonstração do teorema acima, temos que existe $c \in \dot{K}$ tal que $q_{L / K}=\langle c, c, \ldots, c\rangle$ e $\operatorname{det}\left(q_{L / K}\right)=c^{n}$. Se $n=[L: K]$ é par, então $\operatorname{det}\left(q_{L / K}\right)$ é um quadrado em $K$. Se $n$ é ímpar, então do teorema 2.8, temos que $L \supseteq K$ admite uma base normal autodual. Neste caso, $c=\operatorname{Tr}_{L / K}\left(a^{2}\right)=q_{L / K}(a)=1 \mathrm{e}$, portanto, $\operatorname{det}\left(q_{L / K}\right)=1$, que é um quadrado em $K$.

O próximo resultado nos mostra a não existência de base normal auto-dual para extensões galoisianas de corpos de grau 2.

Lema 3.8 Seja $L \supseteq K$ uma extensão galoisiana de grau 2. Então L não admite base normal ortogonal sobre $K$.

Demonstração: Suponhamos que $L \supseteq K$ admite uma base normal ortogonal. Como $L \supseteq K$ é uma extensão quadrática e galoisiana, do lema 3.2 temos que, $q_{L / K}=\langle 2,2 b\rangle$, onde $b \in \dot{K}$, não é um quadrado e $L=K(\sqrt{b})$. Assim, $\operatorname{det}\left(q_{L / K}\right)=4 b$ que não é um quadrado em $K$, o que contradiz o corolário 3.7 .

Nos próximos três resultados, estaremos apresentando as relações entre a existência de bases normais ortogonais e/ou auto-duais, de extensões galoisianas de $\operatorname{corpos} L \supseteq K$, 
com a existência de tais bases para extensões $F \supseteq K$, onde $F$ é um corpo intermediário. Recordemos que se $H$ é um subgrupo normal de $\operatorname{Gal}(L / K)$, então $L^{H}$ denota o subcorpo de $L$ fixado por $H$, ou seja $L^{H}=\{x \in L ; \sigma(x)=x$, para todo $\sigma \in H\}$.

Lema 3.9 Seja $L \supseteq K$ uma extensão galoisiana de corpos com grupo de Galois $G$. Para $H$ subgrupo normal de $G$ tal que, $C a r(K)$ não divide $[G: H], F=L^{H}$ e $y \in F$, temos:

(a) Existe $y^{\prime} \in L$, tal que $y=\sum_{\phi \in H} \phi\left(y^{\prime}\right)$.

(b) Para $y^{\prime} \in L$, como no item anterior, temos que $T^{\prime}(x, y)=T\left(x, y^{\prime}\right)$ onde $T^{\prime}(x, y)=$ $\operatorname{Tr}_{F / K}(x y)$ e $T\left(x, y^{\prime}\right)=\operatorname{Tr}_{L / K}\left(x y^{\prime}\right)$, para todo $x \in F$.

Demonstração: Se $y \in F$, basta considerarmos $y^{\prime}=\frac{y}{m}$, onde $m=[G: H]$. De fato,

$$
\begin{gathered}
\phi_{1}\left(y^{\prime}\right)+\phi_{2}\left(y^{\prime}\right)+\cdots+\phi_{m}\left(y^{\prime}\right)= \\
\phi_{1}\left(\frac{y}{m}\right)+\phi_{2}\left(\frac{y}{m}\right)+\cdots+\phi_{m}\left(\frac{y}{m}\right)= \\
\frac{y}{m}+\cdots+\frac{y}{m}=y
\end{gathered}
$$

mostrando assim o item (a).

Para mostrarmos (b) consideremos $G=\operatorname{Gal}(L / K)=\left\{\sigma_{1}, \ldots, \sigma_{n}\right\}$, e $G / H \cong$ $\left\{\rho_{1}, \cdots, \rho_{m}\right\} \cong \operatorname{Gal}(F / K)$ o grupo quociente. Assim para todo $x, y \in F$, temos que

$$
\begin{aligned}
T^{\prime}(x, y)=\operatorname{Tr}_{F / K}(x y) & =\operatorname{Tr}_{F / K}\left(x \sum_{\phi \in H} \phi\left(y^{\prime}\right)\right)= \\
\sum_{\rho \in G / H} \rho(x) \rho\left(\sum_{\phi \in H} \phi\left(y^{\prime}\right)\right) & =\sum_{\rho \in G / H} \sum_{\phi \in H}(\rho \phi)(x)(\rho \phi)\left(y^{\prime}\right)= \\
\sum_{\sigma \in G} \sigma(x) \sigma\left(y^{\prime}\right) & =\operatorname{Tr}_{L / K}\left(x y^{\prime}\right)=T\left(x, y^{\prime}\right) .
\end{aligned}
$$

Lema 3.10 Se $L \supseteq K$ é uma extensão galoisiana que possui uma base normal autodual sobre $K$ e $H$ é um subgrupo normal de $G=\operatorname{Gal}(L / K)$, tal que, $\operatorname{Car}(K)$ não divide $[G: H]$, então: 
(a) A forma traço $q_{F / K}$, de $F=L^{H}$ sobre $K$ é isométrica a forma $\langle 1,1, \ldots, 1\rangle$, $[F: K]$-vezes.

(b) A extensão galoisiana $L^{H}=F \supseteq K$ admite uma base normal auto-dual.

Demonstração: Sejam $a \in L$ um gerador da base normal de $L$ sobre $K$ e $G / H \cong$ $\left\{\rho_{1}, \ldots, \rho_{m}\right\}$ o grupo quociente. Para cada $\rho \in G / H$, definimos

$$
a_{\rho}=\sum_{\phi \in H} \phi \rho(a)
$$

Agora o resultado do item (a) segue imediatamente do fato que a família $\left(a_{\rho}\right)_{\rho \in G / H}$ é uma base auto-dual de $F$ sobre $K$ com relação a forma traço $T^{\prime}$ de $F$ sobre $K$, pois, para cada $\rho, \rho^{\prime} \in G / H$, usando o lema anterior, obtemos que

$$
T^{\prime}\left(a_{\rho}, a_{\rho^{\prime}}\right)=T\left(a_{\rho}, \rho^{\prime}(a)\right) \stackrel{(\star)}{=} \sum_{\phi \in H} T\left(\phi \rho(a), \rho^{\prime}(a)\right)=\sum_{\phi \in H} \delta_{\phi \rho, \rho^{\prime}}=\delta_{\rho, \rho^{\prime}}
$$

Finalmente, o resultado do item (b) segue do fato que o elemento $a_{1}=\sum_{\phi \in H} \phi(a)$ é um gerador de uma base normal auto-dual de $F$ sobre $K$, ou seja, $\left\{\rho\left(a_{1}\right) ; \rho \in G / H\right\}$ é uma base auto-dual de $F$ sobre $K$.

Do fato de $H$ ser normal em $G$, temos que para cada $\rho \in G / H, \rho\left(a_{1}\right)=\sum_{\phi \in H} \rho \phi(a)=$ $\sum_{\phi \in H} \phi \rho^{\prime}(a)$, para algum $\rho^{\prime} \in G / H$. De $(\star)$, temos que $\rho\left(a_{1}\right)=a_{\rho^{\prime}}$, mostrando assim que $\left\{\rho\left(a_{1}\right) ; \rho \in G / H\right\}=\left(a_{\rho}\right)_{\rho \in G / H}$. Agora o resultado segue da demonstração do item (a).

\section{Demonstração do Teorema 3.1, item (a)}

Queremos mostrar que se $G$ é um grupo finito de ordem par que possui um subgrupo $H$ de índice 2 , então $L \supseteq K$ não admite base normal ortogonal para qualquer extensão de corpos $L \supseteq K$ com grupo de Galois $G$. Faremos o caso mais geral, substituindo base normal ortogonal, por base normal auto-dual. 
Suponhamos que existe uma extensão galoisiana de corpos $L \supseteq K \operatorname{com} G=$ $\operatorname{Gal}(L / K)$ tal que $L$ admite uma base normal auto-dual sobre $K$. Desde que $H$ é em subgrupo de $G$ de índice 2, temos que $H$ é um subgrupo normal de $G$ e, conseqüentemente $H=\operatorname{Gal}\left(L^{H} / K\right)$, ou seja, $L^{H} \supseteq K$ é uma extensão galoisiana de grau 2.

Temos então as extensões galoisianas de corpos $L \supseteq L^{H} \supseteq K$ com $L \supseteq K$ admitindo uma base normal auto-dual. Desde que $\operatorname{Car}(K) \neq 2$, temos que esta não divide $[G: H]$, do lema 3.10 temos que $L^{H} \supseteq K$ admite uma base normal auto-dual, o que contradiz o lema 3.8 , pois $\left[L^{H}: K\right]=2$, mostrando assim o item (a).

\section{Demonstração do Teorema 3.1, item (b)}

No que segue, nos dedicaremos a demonstração do ítem (b) do teorema 3.1, ou seja, mostrar que se $G$ é um grupo de ordem par e seu 2 subgrupo de Sylow é abeliano, então existe uma extensão galoisiana de $\operatorname{corpos} L \supseteq K$ com grupo de Galois $G$ tal que $L$ não admite uma base normal ortogonal sobre $K$.

Dizemos que $G$ é um 2-grupo se sua ordem for uma potência de 2 . O expoente de um grupo finito $G$ é o menor inteiro positivo $n$ tal que $\sigma^{n}=1$, para todo $\sigma \in G$. Observe que o expoente de um 2-grupo é obrigatoriamente uma potência de 2.

Vamos mostrar agora que nenhuma extensão de corpos $L \supseteq K$ com grupo de Galois $G$, sendo $G$ um 2-grupo abeliano e com expoente $d$, e $K$ contendo uma $d$-ésima raiz primitiva da unidade, possui a forma traço $q_{L / K}$ hiperbólica.

De fato, seja $G$ um 2-grupo abeliano. Da decomposição dos grupos abelianos finitos, temos que $G \cong \mathbb{Z}_{n_{1}} \times \mathbb{Z}_{n_{2}} \times \cdots \times \mathbb{Z}_{n_{r}}$ onde $n_{i+1} \mid n_{i}$, para cada $i=1, \cdots, r-1 \mathrm{e}$ $|G|=n_{1} n_{2} \cdots n_{r}$. Observe que cada $n_{i}$ é uma potência de 2. Consideremos o caso $r=2$. Para obtermos o caso geral, basta aplicarmos o processo de indução finita sobre $r$.

Sejam $K_{1}$ um corpo contendo as raízes $n_{i}$-ésimas primitivas da unidade, para $i=$ 
$1,2, K=K_{1}\left(a_{1}, a_{2}\right)$ e $L=K_{1}\left(\sqrt[n_{1}]{a_{1}}, \sqrt[n_{2}]{a_{2}}\right)$, onde $a_{1}$ e $a_{2}$ são variáveis algebricamente independentes sobre $K_{1}$.

Para $F=K\left(\sqrt[n_{1}]{a_{1}}\right)$, temos $K \subseteq F \subseteq L$. Do lema 3.4 temos que $q_{F / K}=\left\langle n_{1}, n_{1} a_{1}\right\rangle$ e $q_{L / F}=\left\langle n_{2}, n_{2} a_{2}\right\rangle$. Mais ainda, do teorema 1.16, temos que $q_{L / K}=\left\langle n_{1}, n_{1} a_{1}\right\rangle \otimes$ $\left\langle n_{2}, n_{2} a_{2}\right\rangle=\left\langle n_{1} n_{2}\right\rangle \otimes\left(\left\langle 1, a_{1}\right\rangle \otimes\left\langle 1, a_{2}\right\rangle\right)=\langle|G|\rangle \otimes\left(\left\langle 1, a_{1}\right\rangle \otimes\left\langle 1, a_{2}\right\rangle\right)$. Esta forma é anisotrópica e conseqüentemente não-hiperbólica sobre $K_{1}\left(a_{1}, a_{2}\right)$.

Mostraremos que de fato $q_{L / K}$ é anisotrópica. Note que

$$
\left\langle 1, a_{1}\right\rangle \otimes\left\langle 1, a_{2}\right\rangle=\left(\langle 1\rangle \otimes\left\langle 1, a_{1}\right\rangle\right) \perp\left(\left\langle a_{2}\right\rangle \otimes\left\langle 1, a_{1}\right\rangle\right)=\left\langle 1, a_{1}\right\rangle \otimes\left\langle a_{2}\right\rangle\left\langle 1, a_{1}\right\rangle .
$$

Se $q_{L / K}$ for isotrópica, existirá $x \in \dot{L}$, tal que $q_{L / K}(x)=0$. Assim, $\left\langle 1, a_{1}\right\rangle(x) \perp$ $\left\langle a_{2}\right\rangle\left\langle 1, a_{1}\right\rangle(x)=0$ e, conseqüentemente, $\left\langle 1, a_{1}\right\rangle(x)=-\left\langle a_{2}\right\rangle\left\langle 1, a_{1}\right\rangle(x)$.

Note que do lado esquerdo da última igualdade temos um polinômio $p\left(a_{2}\right)$ com grau digamos $m$. Do lado direito da igualdade temos um outro polinômio $q\left(a_{2}\right)$ com grau $m+1$, o que leva a uma contradição. Usando isso e um processo indutivo, obtemos

Lema 3.11 Sejam $G \cong \mathbb{Z}_{n_{1}} \times \cdots \times \mathbb{Z}_{n_{r}}$ um 2-grupo abeliano, $K=K_{1}\left(a_{1}, \ldots, a_{r}\right)$ e $L=K_{1}\left(\sqrt[n_{1}]{a_{1}}, \ldots, \sqrt[n_{r}]{a_{r}}\right)$ onde $K_{1}$ é um corpo contendo as $n_{i}$-ésimas raízes primitivas da unidade, com $i=1,2, \cdots, r$, e $a_{1}, a_{2}, \ldots, a_{n}$ são variáveis algebricamente independentes sobre $K_{1}$. Então $q_{L / K}$ não é hiperbólica sobre $K$.

Sejam $H$ um 2-subgrupo de Sylow de $G$ abeliano, $m=[G: H]$ e $|G|=2^{r} m$, para $\operatorname{algum} r \in \mathbb{N}$.

Lema 3.12 Existe uma única forma quadrática $q^{\prime}$, de dimensão $2^{r}$, tal que $q_{L / K} \simeq$ $\langle m\rangle \otimes q^{\prime}$, isto é, $q_{L / K}$ é isométrica à soma direta ortogonal de $m$ cópias de $q^{\prime}$. Esta forma é única a menos de isometria.

Demonstração: A existência segue do resultado 6.1 em [3]. Mostraremos a unicidade. Se $q_{L / K} \simeq\langle m\rangle \otimes q^{\prime \prime}$, então $\langle m\rangle \otimes\left(q^{\prime}-q^{\prime \prime}\right)=0$ em $W(K)$, o que implica que $q^{\prime} \simeq q^{\prime \prime}$, pois $W(K)$ não possui divisores de zero de dimensão ímpar, ver corolário 2.6.5 em [11]. 
Iremos utilizar o seguinte resultado sobre o comportamento de formas quadráticas sobre extensões transcendentes.

Lema 3.13 Sejam F um corpo e q uma forma quadrática sobre F. Se q é anisotrópica sobre $F$, então q também será anisotrópica sobre $F(X)$, o corpo de frações do anel de polinômios em uma variável $X$, sobre $F$.

Demonstração: Como $\operatorname{Car}(F) \neq 2$, temos que $q$ é da forma $\left\langle a_{1}, a_{2}, \cdots, a_{n}\right\rangle$, com $a_{i} \in$ $\dot{F}$, para todo $i=1,2, \cdots, n$. Se $q$ é isotrópica sobre $F(X)$, então, multiplicando por um polinômio conveniente, se necessário, temos que existem polinômios $f_{i}(X) \in F(X)$, não todos nulos, tais que $\sum_{i=1}^{n} a_{i} f_{i}(X)^{2}=0$. Mais ainda, podemos assumir, sem perda de generalidade, que $X$ não divide todos os polinômios $f_{i}(X)$. Em particular, tomando $X=0$, obtemos $\sum_{i=1}^{n} a_{i} f_{i}(0)^{2}=0$, onde $f_{i}(0) \in F$ são não todos nulos. Portanto, $q$ é isotrópica sobre $F$, o que contradiz a hipótese.

Para atingirmos o nosso objetivo final, iremos relacionar formas quadráticas hiperbólicas com representações lineares fiéis de um grupo fnito $G$.

Uma representação de um grupo $G$ em um espaço vetorial $V$ sobre um corpo $K$ é um homomorfismo de grupos $\Phi: G \rightarrow G L(V)=\{f: V \rightarrow V ; \quad f \quad$ é $K-$ automorfismo\}, isto é, uma representação é uma aplicação $\Phi: G \rightarrow G L(V)$ tal que $\Phi\left(\sigma_{1} \sigma_{2}\right)=\Phi\left(\sigma_{1}\right) \Phi\left(\sigma_{2}\right)$, para todo $\sigma_{1}, \sigma_{2} \in G$.

Neste caso, $V$ é chamado o espaço de representação e a dimensão de $V$ é chamada a dimensão da representação. Uma representação de grupo é dita ser uma representação fiel se ela for injetora. Observe que para falarmos de uma representação de um grupo, devemos exibir o espaço vetorial $V$ e o homomorfismo. Algumas vezes, neste trabalho, por abuso de notação, denotaremos a representação de um grupo, apenas por $V$.

Denotaremos também $K(V) \supseteq K$ como uma extensão de corpos, onde $K(V)=$ $K\left(\Psi\left(\sigma_{1}\right), \cdots, \Psi\left(\sigma_{n}\right)\right)$ e $\Psi$ é a representação do grupo $G=\left\{\sigma_{1}, \cdots, \sigma_{n}\right\}$ sobre o $K$ espaço vetorial $V$. 
Iremos agora relacionar representações lineares fiéis com as extensões de corpos e suas respectivas formas traços. No próximo resultado, mostraremos que o fato da forma traço ser hiperbólica não depende da escolha de uma representação fiel.

Lema 3.14 Sejam $V$ e $V^{\prime}$ duas representações lineares fiéis de um grupo finito G sobre um corpo $K$, e consideremos $L=K(V), K_{1}=L^{G}, L^{\prime}=K\left(V^{\prime}\right)$ e $K_{1}^{\prime}=L^{\prime} G$. Se $q_{L / K_{1}}$ é hiperbólica, então $q_{L^{\prime} / K_{1}^{\prime}}$ também será.

Demonstração: Sejam $n=\operatorname{dim}_{K}(V), n^{\prime}=\operatorname{dim}_{K}\left(V^{\prime}\right), s=\left(s_{1}, s_{2}, \cdots, s_{n}\right)$ e $t=$ $\left(t_{1}, \ldots, t_{n^{\prime}}\right)$ úplas de variáveis independentes sobre $K$ e $K^{\prime}$, respectivamente. Pelo lema sem nome no apêndice 3 de $[12]$, temos que $L(t) \cong L^{\prime}(s)$ como $K[G]$ álgebras, onde $G$ age trivialmente nas variáveis.

Assim, desde que $q_{L / K_{1}}$ é hiperbólica, temos que $q_{L(t) / K_{1}(t)} \simeq q_{L^{\prime}(s) / K_{1}^{\prime}(s)}$ é hiperbólica sobre $K_{1}(t) \cong K_{1}^{\prime}(s)$. Agora, o resultado segue do fato que a aplicação natural $W\left(K_{1}^{\prime}\right) \rightarrow W\left(K_{1}^{\prime}(s)\right)$ é injetiva. A injetividade segue do lema anterior.

Vamos agora mostrar que sob certas condições, a extensão galoisiana $L$ sobre $K$ não admite uma base normal ortogonal. Feito isso, poderemos enfim, concluir a demonstração do item (b) do teorema 3.1.

Proposição 3.15 Seja $V$ uma representação linear fiel de um grupo G sobre um corpo $K_{1}, L=K_{1}(V), K=L^{G}$, e seja $G_{2} \leq G$ um 2-subgrupo de Sylow abeliano de $G$. Suponha que $n$ é o expoente de $G_{2}$ e que $K$ contém uma raiz n-ésima primitiva da unidade. Então a extensão galoisiana $L$ sobre $K$ não admite uma base normal ortogonal.

Demonstração: Se mostrarmos que $q_{K_{1}(V) / K_{1}(V)^{G_{2}}}=q_{L / L^{G_{2}}}$ não é hiperbólica, então pelo lema 3.12 teremos que $q_{L / K}$ não será hiperbólica, e portanto pelo teorema 3.6 teremos que $L$ sobre $K$ não possui base normal ortogonal e, conseqüentemente, não possui base normal auto-dual. 
Mostraremos então que $q_{L / L^{G_{2}}}$ não é hiperbólica.

Seja $G_{2} \cong \mathbb{Z}_{n_{1}} \times \mathbb{Z}_{n_{2}} \times \cdots \times \mathbb{Z}_{n_{r}}$ um 2-grupo abeliano com expoente $n$. Note que se $\sigma \in G_{2}$ então podemos associar $\sigma$ a uma $r$-upla $\left(\overline{m_{1}}, \ldots, \overline{m_{r}}\right)$ onde $\overline{m_{i}} \in \mathbb{Z}_{n_{i}}$ e $1<m_{i}<n_{i}$, para cada $i=1, \ldots, r$. O subgrupo $G_{2}$ possui uma representação fiel $\Phi$ de dimensão $r$ sobre $K_{1} \times \cdots \times K_{1}=K_{1}^{r}=S$ dada por

$$
\sigma \mapsto \Phi(\sigma)\left(x_{i}\right)=\left(\omega_{n_{i}}\right)^{m_{i}} x_{i}
$$

onde $\omega_{n_{i}}$ é uma raiz $n_{i}$-ésima primitiva da unidade e $x=\left(x_{1}, \ldots, x_{r}\right) \in S$. Mostremos que de fato $\Phi$ é uma representação de $G_{2}$. Note que para $x=\left(x_{1}, \ldots, x_{r}\right) \in S$ e $\sigma, \rho \in G_{2}$, com $\sigma$ associado a $\left(\overline{m_{1}}, \ldots, \overline{m_{r}}\right)$ e $\rho$ associado a $\left(\overline{l_{1}}, \ldots, \overline{l_{r}}\right)$, temos

$$
\begin{gathered}
\Phi(\sigma \rho)(x)=(\sigma \rho)\left(x_{1}, x_{2}, \ldots, x_{r}\right)=\left(\omega_{n_{1}}^{m_{1}+l_{1}} x_{1}, \ldots, \omega_{n_{r}}^{m_{r}+l_{r}} x_{r}\right)= \\
\left(\omega_{n_{1}}^{m_{1}} x_{1}, \ldots, \omega_{n_{r}}^{m_{r}} x_{r}\right)\left(\omega_{n_{1}}^{l_{1}} x_{1}, \ldots, \omega_{n_{r}}^{l_{r}} x_{r}\right)=\Phi(\sigma)(x) \Phi(\rho)(x) .
\end{gathered}
$$

A representação $\Phi$ é de fato fiel, pois se $\Phi(\sigma)=\Phi(\rho)$, então $\left(\omega_{n_{i}}\right)^{m_{i}} x_{i}=\left(\omega_{n_{i}}\right)^{l_{i}} x_{i}$, para cada $i=1,2, \ldots, r$. Conseqüentemente $\left(\omega_{n_{i}}\right)^{m_{i}}=\left(\omega_{n_{i}}\right)^{l_{i}}$, para cada $i=1,2, \ldots, r$. Como $0 \leq m_{i}, l_{i} \leq n_{i}$ e $\omega_{n_{i}}$ é uma $n_{i}$-ésima raiz primitiva da unidade, temos que $m_{i}=l_{i}$ para cada $i=1,2, \ldots, r$, e portanto $\Phi$ é injetora.

Observe que

$$
K_{1}(S)=K_{1}\left(\sqrt[n_{1}]{a_{1}}, \ldots, \sqrt[n_{n}]{a_{r}}\right) \text { e } K_{1}(V)^{G_{2}}=K_{1}\left(a_{1}, \ldots, a_{r}\right)
$$

onde $a_{1}, a_{2}, \ldots, a_{n}$ são variáveis algebricamente independentes sobre $K_{1}$, e $a_{i}=x_{i}^{n_{i}}$, com $i=1, \cdots, r$. De fato, considere $\sigma \in G_{2}$ associado ao elemento $(\overline{1}, \ldots, \overline{1})$, então $\Psi(\sigma)\left(x_{i}\right)=\omega_{n_{i}} x_{i}$, para cada $i=1, \ldots, r$. Portanto, $K_{1}(S)=K_{1}\left(\omega_{n_{1}} x_{1}, \ldots, \omega_{n_{r}} x_{r}\right)$. Além disso, para cada $i=1, \ldots, r$, temos que $\omega_{n_{i}} x_{i}=\sqrt[n_{i}]{x_{i}^{n_{i}}}=\sqrt[n_{i}]{a_{i}}$, pois $a_{i}=x_{i}^{n_{i}}$, portanto a igualdade segue. Pelo lema 3.11, temos que $q_{K_{1}(S) / K_{1}(S)^{G_{2}}}$ não é hiperbólica e conseqüentemente pelo lema 3.14 teremos que $q_{L / L^{G_{2}}}$ também não será hiperbólica.

A proposição demonstrada acima é uma versão mais fraca do teorema 3.1 item (b), pois neste teorema, não garantimos que o corpo $K$ tenha a $n$-ésima raiz primitiva da 
unidade. Logo, para fecharmos a demonstração do item (b) vamos supor agora que $K$ não contenha uma raiz $n$-ésima primitiva da unidade. Seja $\omega$ uma raiz $n$-ésima primitiva da unidade. Consideremos então a extensão de corpos $L(\omega) \supseteq K(\omega)$. Basta mostrarmos que esta extensão é galoisiana pois, feito isso, as hipóteses da proposição 3.15 estarão satisfeitas e portanto o teorema 3.1, item (b), estará provado.

Como a extensão $L \supseteq K$ é galoisiana, temos que $L$ é o corpo de raízes de um polinômio separável $p(X) \in K[X]$. Se $\omega \in L$, então ainda teremos que $L$ será corpo de raízes do polinômio separável $p(X) \in K(\omega)[X]$. Conseqüentemente, a extensão $L=L(\omega)$ sobre $K(\omega)$ é uma extensão galoisiana.

Se $\omega \notin L$, então consideremos $q(X)=p(X)\left(X^{n}-1\right)$. Claramente, temos que $q(X)$ é um polinômio separável em $K(\omega)[X]$ e $L(\omega)$ é o corpo de raízes de $q(X)$ sobre $K(\omega)[X]$. Seja $G=\operatorname{Gal}(L / K)=\left\{\sigma_{1}, \ldots, \sigma_{n}\right\}$. Temos que $\sigma_{i}(x)=x$, para todo $x \in K$, $\operatorname{com} i=1, \ldots, n$.

Consideremos $G^{\prime}=\operatorname{Gal}(L(\omega) / K(\omega))$. Desde que cada elemento de $G^{\prime}$ deixa $\omega$ fixo, temos que $G^{\prime} \cong G$. Temos então que $L(\omega) / K(\omega)$ satisfaz as hipóteses da proposição 3.15, e portanto o teorema 3.1 (b) está provado.

Para compreender melhor esse resultado, vamos apresentar um exemplo.

Exemplo 3.16 Consideremos a extensão de corpos $L \supseteq K$ dada por $L=\mathbb{Z}_{3}(i)$, $K=\mathbb{Z}_{3}$ com $i$ a unidade imaginária. Mostraremos que o teorema 3.1 se aplica neste exemplo, ou seja, a extensão $L$ sobre $K$ não possui base normal ortogonal. Na verdade, mostraremos que todas as possíveis bases normais desta extensão não são ortogonais.

Primeiro, note que o polinômio minimal de $i$ sobre $\mathbb{Z}_{3}$ é o polinômio $p(X)=X^{2}+\overline{1}$. Esse polinômio é irredutível sobre $\mathbb{Z}_{3}$, pois não possui raízes em $\mathbb{Z}_{3}$. Claramente $i$ é a raiz de $p(X)$. Concluímos então que $\left[\mathbb{Z}_{3}(i): \mathbb{Z}_{3}\right]=2, \operatorname{logo}|G|=|\operatorname{Gal}(L / K)|=2$, então $G=\{1, \sigma\}$ com $\sigma(i)=\overline{2} i$. Temos também que $\mathbb{Z}_{3}(i)=\{\overline{0}, \overline{1}, \overline{2}, i, \overline{2} i, \overline{1}+i, \overline{2}+$ $i, \overline{1}+\overline{2} i, \overline{2}+\overline{2} i\}$. 
Analisando todos os possíveis casos, obtemos que as únicas bases normais de $\mathbb{Z}_{3}(i)$ sobre $\mathbb{Z}_{3}$ são $\mathcal{B}_{1}=\{\overline{1}+i, \overline{1}+\overline{2} i\}=\{\overline{1}+i, \sigma(\overline{1}+i)\}$ e $\mathcal{B}_{2}=\{\overline{2}+\overline{2} i, \overline{2}+i\}=$ $\{\overline{2}+\overline{2} i, \sigma(\overline{2}+\overline{2} i)\}$.

Com relação a $\mathcal{B}_{1}$, temos que

$$
\begin{aligned}
T(\overline{1}+i, \overline{1}+i) & =\operatorname{Tr}_{L / K}(\overline{2} i)=\overline{2} i+\sigma(\overline{2} i) \\
& =\overline{2} i+(\overline{-2} i)=\overline{0} \\
T(\overline{1}+i, \overline{1}+\overline{2} i) & =T(\overline{1}+\overline{2} i, \overline{1}+i) \\
& =\operatorname{Tr}_{L / K}(\overline{2})=\overline{2}+\sigma(\overline{2})=1, \\
T(\overline{1}+\overline{2} i, \overline{1}+\overline{2} i) & =\operatorname{Tr}_{L / K}(i)=i+\sigma(i)=i+\overline{2} i=\overline{0},
\end{aligned}
$$

ou seja, $\mathcal{B}_{1}$ é uma base normal não ortogonal.

Com relação a $\mathcal{B}_{2}$, temos

$$
\begin{aligned}
T(\overline{2}+\overline{2} i, \overline{2}+\overline{2} i) & =\operatorname{Tr}_{L / K}(\overline{2} i)=\overline{0} \\
T(\overline{2}+\overline{2} i, \overline{2}+i) & =T(\overline{2}+i, \overline{2}+\overline{2} i)=\operatorname{Tr}_{L / K}(\overline{2})=\overline{1} \\
T(\overline{2}+i, \overline{2}+i) & =\operatorname{Tr}_{L / K}(i)=\overline{0}
\end{aligned}
$$

ou seja, $\mathcal{B}_{2}$ também é uma base normal não ortogonal. 



\section{Referências Bibliográficas}

[1] Bass, H., Unitary algebraic K-theory. Lecture Notes in Mathematics, 343 (1973), $57-265$.

[2] Bayer-Fluckiger, E., Self-dual normal bases, Nedel, Akad. Wetensch, Indag. Math., 51 (1989), no. 4, 379-383.

[3] Bayer-Fluckiger, E., Serre, J. P., Torsions Quadratiques et Bases Normales Autoduales, Amer. J. Math., 116 (1994), 1-64.

[4] Hoffman, K., Kunze, R., Linear Algebra, Prentice-Hall, New Jersey, 2.ed.,(1971).

[5] Jacobson, N., Basic Algebra I, W. H. Freeman and Company, New York (1985).

[6] Kang, D. S., Nonexistence of self-dual normal bases, Comm. in Algebra, 32, no. 1 (2004), 125-132.

[7] Kang, D. S., Reichstein, Z., Trace forms of Galois field extensions in the presence of roots of unity, J. Reine Angew Math., 549 (2002), 79-89.

[8] Lam, T. Y., The Algebraic Theory of Quadratic Forms, University of California (1973).

[9] Lang, S., Algebra, Addison-Wesley, Reading (1965).

[10] Milies, C. P., Anéis e Módulos, IME-USP, Publicações do Instituto de Matemática e Estatística da Universidade de São Paulo (1972).

[11] Scharlau, W., Quadratic and Hermitian Forms, Grundlehren der Math. Wiss. 270, Springer-Verlag (1985).

[12] Shafarevich, I. R., Basic Algebraic Geometry, vol. 1, 2.ed., Springer-Verlag (1994). 